\title{
Rothe method and numerical analysis for history-dependent hemivariational inequalities with applications to contact mechanics
}

\author{
Stanisław Migórski ${ }^{1,2} \cdot$ Shengda Zeng ${ }^{3}$ (D)
}

Received: 4 April 2018 / Accepted: 25 September 2018 / Published online: 23 January 2019

(C) The Author(s) 2019

\begin{abstract}
In this paper, an abstract evolutionary hemivariational inequality with a historydependent operator is studied. First, a result on its unique solvability and solution regularity is proved by applying the Rothe method. Next, we introduce a numerical scheme to solve the inequality and derive error estimates. We apply the results to a quasistatic frictional contact problem in which the material is modeled with a viscoelastic constitutive law, the contact is given in the form of multivalued normal compliance, and friction is described with a subgradient of a locally Lipschitz potential. Finally, for the contact problem, we provide the optimal error estimate.
\end{abstract}

Keywords Hemivariational inequality · Clarke subgradient · History-dependent operator $\cdot$ Rothe method · Finite element method · Error estimates · Viscoelastic material $\cdot$ Frictional contact

Mathematics Subject Classification (2010) 35L15 · 35L86 · 35L87 · 74Hxx · 74M10

Shengda Zeng

zengshengda@163.com; shengdazeng@gmail.com; shdzeng@hotmail.com

Stanisław Migórski

stanislaw.migorski@uj.edu.pl

1 College of Applied Mathematics, Chengdu University of Information Technology, Chengdu, 610225, Sichuan Province, People's Republic of China

2 Chair of Optimization and Control, Jagiellonian University in Krakow, ul. Lojasiewicza 6, 30348 Krakow, Poland

3 Faculty of Mathematics and Computer Science, Jagiellonian University in Krakow, ul. Lojasiewicza 6, 30348 Krakow, Poland 


\section{Introduction}

In this paper, we are concerned with the existence and uniqueness of a solution to an abstract evolutionary hemivariational inequality which involves a history-dependent operator of the form

$$
\left\langle A u^{\prime}(t)+B u(t)+(\mathcal{R} u)(t)-f(t), v\right\rangle+J^{0}(M u(t) ; M v) \geq 0
$$

for all $v \in V$, a.e. $t \in(0, T)$ with $u(0)=u_{0}$. Here, $A$ and $B$ are operators from a reflexive Banach space $V$ to its dual $V^{*}, M$ is a linear, bounded operator, $J^{0}$ denotes the generalized gradient of a locally Lipschitz function, $f:(0, T) \rightarrow V^{*}$ and $u_{0} \in V$ are given, and $\mathcal{R}$ represents a history-dependent operator.

The motivation to study the inequality of the form (1) comes from contact problems in solid mechanics. It is known that when the external forces and tractions evolve slowly in time in such a way that the acceleration in the system is rather small and negligible, then the inertial terms can be neglected. In such a way, we obtain the quasistatic approximation (equilibrium equation) for the equation of motion. Quasistatic contact models have been studied in several monographs and many papers dedicated to such phenomena (see $[9,14,35,36]$ and the references therein).

In the first part of the paper, we deal with an abstract time-dependent hemivariational inequality of the form (1). The main results are delivered on existence, uniqueness, and regularity of a solution to the abstract hemivariational inequality (see Theorem 11). We apply the Rothe method (see [17, 18]), combined with a surjectivity result for a multivalued and coercive operator. The hemivariational inequality (1) without a history-dependent operator has been recently investigated in [24] by using the vanishing acceleration method, where a local existence result was proved. In contrast to Theorem 17 of [24], here, we provide a result on the global unique solvability to (1). Also, our proof is now based on the Rothe method and is simpler, since we have eliminated the additional space $Z$ required in [24]. Moreover, being motivated by applications to contact mechanics in Section 6, the inequality (1) involves a historydependent operator. We recall that the notion of a history-dependent operator is quite recent and it was introduced in [39]. Various problems with history-dependent operators have been studied for the evolution variational and hemivariational inequalities in $[1,6,10,11,20-22,27-30,34,43,44]$, and for the quasistatic problems in [19, $26,37,38,40,41,45]$. Furthermore, we study a fully discrete approximation for the problem (1) which consists in finite difference discretization in time and finite element approximation in the spatial variable. We prove in Theorem 13 the Céa-type error estimate for the hemivariational inequality.

In the second part of the paper, we apply the abstract results to a quasistatic frictional contact model for viscoelastic materials. The process is described by multivalued versions of the nonmonotone normal compliance and friction boundary conditions. We provide the variational formulation of the contact problem for which we deliver a result on its unique weak global solvability. In this way, we improve the local existence result of [24, Theorem 17]. Finally, for the frictionless contact, we establish a result on an optimal error estimate for the fully discrete approximation scheme. Note that results on numerical analysis for hemivariational inequalities can be found in $[3,12,15,16,37]$ and the references therein. 
The outline of the paper is as follows. After recalling the basic notation in Section 2, in Section 3, we formulate the abstract hemivariational inequality with a history-dependent operator. In Section 4, we apply the Rothe method to deliver existence and uniquence result for this inequality. The error estimate of the Céa type for a fully discrete approximation is provided in Section 5. Finally, in Section 6, we illustrate the applicability of our results to the quasistatic frictional contact problem for viscoelastic material.

\section{Preliminaries}

In this section, we recall the basic notation and some results which are needed in the sequel (see $[5,7,8,42]$ ). We use the standard notation for the Lebesgue and Sobolev spaces of functions defined on a finite time interval $[0, T]$ with values in a Banach space. We denote by $\mathcal{L}(E, F)$ the space of linear and bounded operators from a Banach space $E$ to a Banach space $F$ endowed with the usual norm $\|\cdot\|_{\mathcal{L}(E, F)}$. For a subset $S$ of Banach space $\left(E,\|\cdot\|_{E}\right)$, we write $\|S\|_{E}=\sup \left\{\|s\|_{E} \mid s \in S\right\}$.

Let $Y$ be a reflexive Banach space and $\langle\cdot, \cdot\rangle$ denote the duality of $Y$ and $Y^{*}$. A single-valued mapping $A: Y \rightarrow Y^{*}$ is called monotone if $\langle A u-A v, u-v\rangle \geq 0$ for all $u, v \in Y$. An operator $A: Y \rightarrow Y^{*}$ is pseudomonotone if for every sequence $\left\{y_{n}\right\} \subseteq Y$ converging weakly to $y \in Y$ such that $\lim \sup \left\langle A y_{n}, y_{n}-y\right\rangle \leq 0$, we have

$$
\langle A y, y-z\rangle \leq \liminf \left\langle A y_{n}, y_{n}-z\right\rangle \text { for all } z \in Y \text {. }
$$

Note that the operator $A: Y \rightarrow Y^{*}$ is pseudomonotone if and only if the conditions $y_{n} \rightarrow y$ weakly in $Y$ and $\lim \sup \left\langle A y_{n}, y_{n}-y\right\rangle \leq 0$ entail $\lim \left\langle A y_{n}, y_{n}-y\right\rangle=0$ and $A y_{n} \rightarrow A y$ weakly in $Y^{*}$. It is also easy to check that if $A \in \mathcal{L}\left(Y, Y^{*}\right)$ is nonnegative, then it is pseudomonotone.

We recall the notion of the pseudomonotonicity for a multivalued operator.

Definition 1 Let $Y$ be a reflexive Banach space. An operator $T: Y \rightarrow 2^{Y^{*}}$ is pseudomonotone if

(a) for every $v \in Y$, the set $T v \subset Y^{*}$ is nonempty, bounded, closed, and convex,

(b) $T$ is upper semicontinuous from each finite dimensional subspace of $Y$ to $Y^{*}$ endowed with the weak topology,

(c) for any sequences $\left\{u_{n}\right\} \subset Y$ and $\left\{u_{n}^{*}\right\} \subset Y^{*}$ such that $u_{n} \rightarrow u$ weakly in $Y$, $u_{n}^{*} \in T u_{n}$ for all $n \geq 1$ and $\lim \sup \left\langle u_{n}^{*}, u_{n}-u\right\rangle \leq 0$, we have that for every $v \in Y$, there exists $u^{*}(v) \in T u$ such that

$$
\left\langle u^{*}(v), u-v\right\rangle \leq \liminf _{n \rightarrow \infty}\left\langle u_{n}^{*}, u_{n}-v\right\rangle
$$

We recall the following fundamental surjectivity theorem (see [8, Theorem 1.3.70] or [42]), which will be used to prove existence of a solution to a static hemivariational inequality in Section 4. 
Theorem 2 Let $Y$ be a reflexive Banach space and $T: Y \rightarrow 2^{Y^{*}}$ be pseudomonotone and coercive. Then, $T$ is surjective, i.e., for every $f \in Y^{*}$, there is $u \in Y$ such that $T u \ni f$.

We hereafter recall the definition of the Clarke subgradient.

Definition 3 Given a locally Lipschitz function $J: E \rightarrow \mathbb{R}$ on a Banach space $E$, we denote by $J^{0}(u ; v)$ the generalized (Clarke) directional derivative of $J$ at the point $u \in E$ in the direction $v \in E$ defined by

$$
J^{0}(u ; v)=\limsup _{\lambda \rightarrow 0^{+}, w \rightarrow u} \frac{J(w+\lambda v)-J(w)}{\lambda} .
$$

The generalized gradient of $J: E \rightarrow \mathbb{R}$ at $u \in E$ is defined by

$$
\partial J(u)=\left\{\xi \in E^{*} \mid J^{0}(u ; v) \geq\langle\xi, v\rangle \text { for all } v \in E\right\} .
$$

The following result provides an example of a multivalued pseudomonotone operator which is a superposition of the Clarke subgradient with a compact operator. The proof can be found in [3, Proposition 5.6].

Proposition 4 Let $V$ and $X$ be reflexive Banach spaces, $M: V \rightarrow X$ be a linear, bounded, and compact operator. We denote by $M^{*}: X^{*} \rightarrow V^{*}$ the adjoint operator of $M$. Let $J: X \rightarrow \mathbb{R}$ be a locally Lipschitz function such that

$$
\|\partial J(v)\|_{X^{*}} \leq c\left(1+\|v\|_{X}\right) \text { for all } v \in X
$$

with $c>0$. Then, the multivalued operator $F: V \rightarrow 2^{V^{*}}$ defined by $F(v)=$ $M^{*} \partial J(M v)$ for $v \in V$ is pseudomonotone.

We conclude this section with a discrete version of the Gronwall inequality whose proof can be found in [14, Lemma 7.25].

Lemma 5 Let $T>0$ be given. For a positive integer $N$, we define $\tau=\frac{T}{N}$. Assume that $\left\{g_{n}\right\}_{n=1}^{N}$ and $\left\{e_{n}\right\}_{n=1}^{N}$ are two sequences of nonnegative numbers satisfying

$$
e_{n} \leq \bar{c} g_{n}+\bar{c} \tau \sum_{j=1}^{n-1} e_{j} \text { for } n=1, \ldots, N
$$

for a positive constant $\bar{c}$ independent of $N$ (or $\tau)$. Then, there exists a positive constant $c$, independent of $N$ (or $\tau)$ such that

$$
e_{n} \leq c\left(g_{n}+\tau \sum_{j=1}^{n-1} g_{j}\right) \text { for } n=1, \ldots, N
$$




\section{History-dependent hemivariational inequalities}

In this section, we introduce a class of history-dependent hemivariational inequalities. This class will be studied in Section 4 where the existence and uniqueness result for this class of inequalities will be provided. A fully discrete approximation for the inequalities in this class will be discussed in Section 5.

We use the following standard notation (see [7, 8, 29, 42] for details). Let $V \subset$ $H \subset V^{*}$ be an evolution triple of spaces. Recall that this means that $V$ is a reflexive and separable Banach space, $H$ is a separable Hilbert space, and the embedding $V \subset$ $H$ is dense and continuous. Let $i$ be the embedding operator between $V$ and $H$ which is assumed to be compact. It is known that the adjoint operator $i^{*}: H \rightarrow V^{*}$ is also linear, continuous and compact. The duality pairing between $V^{*}$ and $V$ and a norm in $V$ are denoted by $\langle\cdot, \cdot\rangle$ and $\|\cdot\|$, respectively. For the Hilbert space $H$, we denote its scalar product and a norm by $(\cdot, \cdot)$ and $\|\cdot\|_{H}$, respectively.

Given $0<T<+\infty$, let $\mathcal{V}=L^{2}(0, T ; V)$ and $\mathcal{H}=L^{2}(0, T ; H)$. It follows from the reflexivity of $V$ that both $\mathcal{V}$ and its dual space $\mathcal{V}^{*}=L^{2}\left(0, T ; V^{*}\right)$ are reflexive Banach spaces as well. Identifying $\mathcal{H}=L^{2}(0, T ; H)$ with its dual, we have the continuous embeddings $\mathcal{V} \subset \mathcal{H} \subset \mathcal{V}^{*}$.

The notation $\langle\cdot, \cdot\rangle^{*} \times \mathcal{V}$ stands for the duality pairing between $\mathcal{V}$ and $\mathcal{V}^{*}$. Moreover, by $C(0, T ; V)$, we denote the space of continuous functions on $[0, T]$ with values in $V$.

Let $X$ be a separable and reflexive Banach space. Given operators $A, B: V \rightarrow$ $V^{*}, M: V \rightarrow X$, the function $J: X \rightarrow \mathbb{R}, f \in \mathcal{V}^{*}$ and $u_{0} \in V$, we consider the following evolutionary hemivariational inequality involving a history-dependent operator.

Problem 6 Find an element $u \in \mathcal{V}$ such that $u^{\prime} \in \mathcal{V}$ and

$$
\left\{\begin{array}{l}
\left\langle A u^{\prime}(t)+B u(t)+(\mathcal{R} u)(t)-f(t), v\right\rangle+J^{0}(M u(t) ; M v) \geq 0 \\
\text { for all } v \in V, \text { a.e. } t \in(0, T), \\
u(0)=u_{0} .
\end{array}\right.
$$

Here, $\mathcal{R}: C(0, T ; V) \rightarrow C\left(0, T ; V^{*}\right)$ is an operator defined by

$$
(\mathcal{R} u)(t)=E\left(\int_{0}^{t} q(t, s) u(s) d s+\alpha\right) \text { for } t \in[0, T]
$$

where $E: V \rightarrow V^{*}, \alpha \in V$ and $q:[0, T] \times[0, T] \rightarrow \mathcal{L}(V, V)$.

We impose the following assumptions on the data of Problem 6.

$\underline{H(A)}$ : The operator $A: V \rightarrow V^{*}$ is linear, bounded, coercive, and symmetric, i.e.,

(i) $\quad A \in \mathcal{L}\left(V, V^{*}\right)$.

(ii) $\langle A v, v\rangle \geq m_{A}\|v\|^{2}$ for all $v \in V$ with $m_{A}>0$.

(iii) $\langle A v, w\rangle=\langle A w, v\rangle$ for all $v, w \in V$. 
$\underline{H(B)}$ : The operator $B: V \rightarrow V^{*}$ is linear, bounded, and coercive, i.e.,

(i) $B \in \mathcal{L}\left(V, V^{*}\right)$.

(ii) $\langle B v, v\rangle \geq m_{B}\|v\|^{2}$ for all $v \in V$ with $m_{B}>0$.

$H(E): \quad E \in \mathcal{L}\left(V, V^{*}\right)$.

$\overline{H(q)}$ : The function $q \in C([0, T] \times[0, T], \mathcal{L}(V, V))$ is Lipschitz continuous with respect to the first variable, i.e., there exists $L_{q}>0$ such that

$$
\left\|q\left(t_{1}, s\right)-q\left(t_{2}, s\right)\right\| \leq L_{q}\left|t_{1}-t_{2}\right| \text { for all } t_{1}, t_{2}, s \in[0, T] .
$$

$H(J)$ : The functional $J: X \rightarrow \mathbb{R}$ is such that

(i) $J$ is locally Lipschitz.

(ii) There exists $c_{J}>0$ such that $\|\partial J(u)\|_{X^{*}} \leq c_{J}\left(1+\|u\|_{X}\right)$ for all $u \in X$.

(iii) There exists $m_{J} \geq 0$ such that

$$
\langle\xi-\eta, u-v\rangle_{X^{*} \times X} \geq-m_{J}\|u-v\|_{X}^{2},
$$

for all $u, v \in X$ and $\xi \in \partial J(u), \eta \in \partial J(v)$.

$H(f): \quad f \in \mathcal{V}^{*}$.

$\overline{H(M)}$ : The operator $M: V \rightarrow X$ is linear, continuous, and compact.

$\underline{\left(H_{0}\right)}: \quad m_{B}>m_{J}\|M\|^{2}$.

Remark 7 Hypothesis $H(J)$ (iii) is called the relaxed monotonicity condition for a locally Lipschitz function $J$. It was used in the literature (cf. [23, Section 3.3]) to ensure the uniqueness of the solution to hemivariational inequalities. This hypothesis has the equivalent formulation as follows

$$
J^{0}(u ; v-u)+J^{0}(v ; u-v) \leq m_{J}\|u-v\|_{X}^{2},
$$

for all $u, v \in X$. In addition, examples of nonconvex functions which satisfy the relaxed monotonicity condition can be found in [23, 37]. Particularly, it can be proved that for a convex function, condition $H(J)$ (iii) holds with $m_{J}=0$.

We recall (cf. [39]) that an operator $\mathcal{S}: C(0, T ; V) \rightarrow C\left(0, T ; V^{*}\right)$ is called a history-dependent operator if there exists $L>0$ such that

$$
\left\|\left(\mathcal{S} u_{1}\right)(t)-\left(\mathcal{S} u_{2}\right)(t)\right\|_{V^{*}} \leq L \int_{0}^{t}\left\|u_{1}(s)-u_{2}(s)\right\|_{V} d s
$$

for all $u_{1}, u_{2} \in C(0, T ; V)$ and all $t \in[0, T]$. We remark that under hypotheses $H(E), H(q)$ and $\alpha \in V$, the operator $\mathcal{R}$ defined in (2) satisfies condition (3) with $L=c_{E} c_{q}$, where $c_{E}=\|E\|$ and $c_{q}=\max _{(t, s) \in[0, T] \times[0, T]}\|q(t, s)\|$.

\section{Rothe method}

In this section, we present a result on existence and uniqueness of solution for Problem 6. The technique of proof relies on the Rothe method (known also as a method 
of lines, see [17, 18]). It consists in a time discretization in which we define an approximate sequence of functions by using the implicit (backward) Euler formula. Next, in each time step, we will solve a stationary hemivariational inequality. Finally, we construct the piecewise constant and piecewise affine interpolants and prove a convergence result.

In the rest of the section, we denote by $C>0$ a constant whose value may change from line to line.

Let $N \in \mathbb{N}$ be fixed and denote $f_{\tau}^{k}=\frac{1}{\tau} \int_{t_{k-1}}^{t_{k}} f(s) d s$ for $k=1, \ldots, N$, where $t_{k}=k \tau$ and $\tau=\frac{T}{N}$. Now, we discuss the following discretized problem called the Rothe problem.

Problem 8 Find $\left\{u_{\tau}^{k}\right\}_{k=0}^{N} \subset V$ such that $u_{\tau}^{0}=u_{0}$ and

$$
\left\langle A u_{\tau}^{k}+\tau B u_{\tau}^{k}+\tau x_{\tau}^{k}, v\right\rangle+\tau\left\langle\partial J\left(M u_{\tau}^{k}\right), M v\right\rangle_{X^{*} \times X} \ni\left\langle\tau f_{\tau}^{k}+A u_{\tau}^{k-1}, v\right\rangle,
$$

for all $v \in V$ and for $k=1,2, \ldots, N$, where $x_{\tau}^{k} \in V^{*}$ is defined by

$$
x_{\tau}^{k}=E\left(\alpha+\sum_{j=1}^{k} \int_{t_{j-1}}^{t_{j}} q\left(t_{k}, s\right) u_{\tau}^{j} d s\right) \text {. }
$$

First, we shall prove the existence and uniqueness of a solution to Problem 8.

Lemma 9 Assume that $u_{0} \in V, H(A), H(B), H(E), H(q), H(J), H(M)$, and $\left(H_{0}\right)$ hold. Then, there exists $\tau_{0}>0$ such that, for all $\tau \in\left(0, \tau_{0}\right)$, Problem 8 has a unique solution.

Proof Let $u_{\tau}^{0}, u_{\tau}^{1}, \ldots, u_{\tau}^{k-1}$ be given. We will prove that there exists a unique element $u_{\tau}^{k} \in V$ which satisfies inclusion (4). To end this, we apply Theorem 2 to show that the operator $L: V \rightarrow 2^{V^{*}}$ defined by

$$
L v=A v+\tau B v+\tau E\left(\int_{t_{k-1}}^{t_{k}} q\left(t_{k}, s\right) v d s\right)+\tau M^{*} \partial J(M v)
$$

for all $v \in V$ is surjective.

First, we show that there exists $\tau_{0}>0$ such that, for all $\tau \in\left(0, \tau_{0}\right), L$ is a pseudomonotone operator. Indeed, by hypotheses $H(A)(\mathrm{i})-(\mathrm{ii}), H(B)(\mathrm{i})-(\mathrm{ii}), H(E)$, and $H(q)$, we can easily get that the operator

$$
v \mapsto A v+\tau B v+\tau E\left(\int_{t_{k-1}}^{t_{k}} q\left(t_{k}, s\right) v d s\right)
$$

is bounded, continuous, and monotone for $\tau \in\left(0, \tau_{0}\right)$, where $\tau_{0}=\frac{m_{B}}{c_{E} c_{q}}$ with $c_{E}=$ $\|E\|$ and $c_{q}=\max _{(t, s) \in[0, T] \times[0, T]}\|q(t, s)\|$. From [23, Theorem 3.69], we conclude that the operator defined by (5) is pseudomonotone. On the other hand, taking into account assumptions $H(J)(\mathrm{i})-$ (ii) and $H(M)$ and Proposition 4, it is clear that the operator $v \mapsto M^{*} \partial J(M v)$ is pseudomonotone as well. Therefore, by using [23, Proposition 3.59(ii)], we infer that $L$ is a pseudomonotone operator too. 
Subsequently, we prove that the operator $L$ is coercive. From hypothesis $H(J)$, we derive the estimate (see [12])

$$
\langle\partial J(u), u\rangle_{X^{*} \times X} \geq-m_{J}\|u\|_{X}^{2}-c_{J}\|u\|_{X}
$$

for all $u \in X$. This inequality together with $H(A)(\mathrm{ii}), H(B)(\mathrm{ii}), H(E)$, and $H(q)$ implies

$$
\begin{aligned}
& \left\langle A u+\tau B u+\tau E\left(\int_{t_{k-1}}^{t_{k}} q\left(t_{k}, s\right) u d s\right)+\tau M^{*} \partial J(M u), u\right\rangle \\
& \geq m_{A}\|u\|^{2}+\tau m_{B}\|u\|^{2}-\tau^{2} c_{E} c_{q}\|u\|^{2}-\tau m_{J}\|M\|^{2}\|u\|^{2}-\tau c_{J}\|M\|\|u\| \\
& \quad \geq\left(m_{A}+\tau\left(m_{B}-m_{J}\|M\|^{2}-\tau c_{E} c_{q}\right)\right)\|u\|^{2}-\tau c_{J}\|M\|\|u\|
\end{aligned}
$$

for all $u \in V$. From the smallness condition $\left(H_{0}\right)$, we choose $\tau_{0}=\frac{m_{B}-m_{J}\|M\|^{2}}{c_{E} c_{q}}>0$. Hence, we deduce that the operator $L$ is coercive for all $\tau \in\left(0, \tau_{0}\right)$. Therefore, by the use of Theorem 2, we obtain that $L$ is surjective, i.e., Problem 8 has at least one solution $u_{\tau}^{k} \in V$.

For uniqueness part, we assume that $\bar{u}_{\tau}^{k}$ and $\tilde{u}_{\tau}^{k}$ are two solutions in $V$ of Problem 8, that is,

$$
\left\langle A \bar{u}_{\tau}^{k}+\tau B \bar{u}_{\tau}^{k}+\tau \bar{x}_{\tau}^{k}+\tau M^{*} \partial J\left(M \bar{u}_{\tau}^{k}\right), v\right\rangle \geq\left\langle\tau f_{\tau}^{k}+A u_{\tau}^{k-1}, v\right\rangle \text { for all } v \in V
$$

and

$$
\left\langle A \widetilde{u}_{\tau}^{k}+\tau B \widetilde{u}_{\tau}^{k}+\tau \widetilde{x}_{\tau}^{k}+\tau M^{*} \partial J\left(M \widetilde{u}_{\tau}^{k}\right), v\right\rangle \geq\left\langle\tau f_{\tau}^{k}+A u_{\tau}^{k-1}, v\right\rangle \text { for all } v \in V,
$$

where the elements $\bar{x}_{\tau}^{k}$ and $\tilde{x}_{\tau}^{k}$ are defined by

$$
\bar{x}_{\tau}^{k}=E\left(\alpha+\sum_{j=1}^{k-1} \int_{t_{j-1}}^{t_{j}} q\left(t_{k}, s\right) u_{\tau}^{j} d s+\int_{t_{k-1}}^{t_{k}} q\left(t_{k}, s\right) \bar{u}_{\tau}^{k} d s\right)
$$

and

$$
\tilde{x}_{\tau}^{k}=E\left(\alpha+\sum_{j=1}^{k-1} \int_{t_{j-1}}^{t_{j}} q\left(t_{k}, s\right) u_{\tau}^{j} d s+\int_{t_{k-1}}^{t_{k}} q\left(t_{k}, s\right) \tilde{u}_{\tau}^{k} d s\right),
$$

respectively. We take $v=\widetilde{u}_{\tau}^{k}-\bar{u}_{\tau}^{k}$ in the first inequality and $v=\bar{u}_{\tau}^{k}-\widetilde{u}_{\tau}^{k}$ in the second one. We add the resulting inequalities to get

$$
\begin{aligned}
& \left\langle A \bar{u}_{\tau}^{k}-A \widetilde{u}_{\tau}^{k}, \bar{u}_{\tau}^{k}-\tilde{u}_{\tau}^{k}\right\rangle+\tau\left\langle B \bar{u}_{\tau}^{k}-B \widetilde{u}_{\tau}^{k}, \bar{u}_{\tau}^{k}-\tilde{u}_{\tau}^{k}\right\rangle+\tau\left\langle\bar{x}_{\tau}^{k}-\tilde{x}_{\tau}^{k}, \bar{u}_{\tau}^{k}-\tilde{u}_{\tau}^{k}\right\rangle \\
& \quad+\tau\left\langle\partial J\left(M \bar{u}_{\tau}^{k}\right)-\partial J\left(M \tilde{u}_{\tau}^{k}\right), M \bar{u}_{\tau}^{k}-M \tilde{u}_{\tau}^{k}\right\rangle_{X^{*} \times X} \leq 0 .
\end{aligned}
$$

Hence,

$$
\left(m_{A}+\tau\left(m_{B}-m_{J}\|M\|^{2}-\tau c_{E} c_{q}\right)\right)\left\|\bar{u}_{\tau}^{k}-\widetilde{u}_{\tau}^{k}\right\|^{2} \leq 0 .
$$

The smallness condition $\left(H_{0}\right)$ guarantees that $\bar{u}_{\tau}^{k}=\tilde{u}_{\tau}^{k}$, which completes the proof of this lemma.

Next, we establish the estimates for the solution of Problem 8. 
Lemma 10 Under assumptions of Lemma 9, there exists $\tau_{0}>0$ and $C>0$ independent of $\tau$, such that for all $\tau \in\left(0, \tau_{0}\right)$, the solution $\left\{u_{\tau}^{k}\right\}_{k=0}^{N} \subset V$ of Problem 8 satisfies

$$
\begin{aligned}
& \max _{k=1,2, \ldots, N}\left\|u_{\tau}^{k}\right\| \leq C, \\
& \sum_{k=1}^{N}\left\|u_{\tau}^{k}-u_{\tau}^{k-1}\right\|^{2} \leq C, \\
& \max _{k=1,2, \ldots, N}\left\|\xi_{\tau}^{k}\right\| X^{*} \leq C, \\
& \tau \sum_{k=}^{N}\left\|\frac{u_{\tau}^{k}-u_{\tau}^{k-1}}{\tau}\right\|^{2} \leq C,
\end{aligned}
$$

where $\xi_{\tau}^{k} \in \partial J\left(M u_{\tau}^{k}\right)$.

Proof We choose $v=u_{\tau}^{k}$ in (4), then use the hypotheses $H(A)$ and $H(B)$ and the equality

$$
2\left\langle A u_{\tau}^{k}-A u_{\tau}^{k-1}, u_{\tau}^{k}\right\rangle=\left\langle A u_{\tau}^{k}, u_{\tau}^{k}\right\rangle-\left\langle A u_{\tau}^{k-1}, u_{\tau}^{k-1}\right\rangle+\left\langle A\left(u_{\tau}^{k}-u_{\tau}^{k-1}\right), u_{\tau}^{k}-u_{\tau}^{k-1}\right\rangle
$$

to get

$$
\begin{aligned}
& \frac{1}{2}\left\langle A u_{\tau}^{k}, u_{\tau}^{k}\right\rangle-\frac{1}{2}\left\langle A u_{\tau}^{k-1}, u_{\tau}^{k-1}\right\rangle+\frac{1}{2}\left\langle A\left(u_{\tau}^{k}-u_{\tau}^{k-1}\right), u_{\tau}^{k}-u_{\tau}^{k-1}\right\rangle+\tau m_{B}\left\|u_{\tau}^{k}\right\|^{2} \\
& \quad-\tau m_{J}\|M\|^{2}\left\|u_{\tau}^{k}\right\|^{2}-c_{J}\|M\|\left\|u_{\tau}^{k}\right\| \leq \tau\left\|x_{\tau}^{k}\right\|_{V^{*}}\left\|u_{\tau}^{k}\right\|+\tau\left\|f_{\tau}^{k}\right\|_{V^{*}}\left\|u_{\tau}^{k}\right\| .
\end{aligned}
$$

Next, the assumptions $H(E)$ and $H(q)$ imply

$$
\tau\left\|x_{\tau}^{k}\right\|_{V^{*}}\left\|u_{\tau}^{k}\right\| \leq \tau^{2} c_{E} c_{q} \sum_{j=1}^{k}\left\|u_{\tau}^{j}\right\|\left\|u_{\tau}^{k}\right\|+\tau c_{E}\|\alpha\|\left\|u_{\tau}^{k}\right\| .
$$

Combining (10) and (11), and using the Cauchy inequality with $\varepsilon>0$, we have

$$
\begin{aligned}
& \frac{1}{2}\left\langle A u_{\tau}^{k}, u_{\tau}^{k}\right\rangle-\frac{1}{2}\left\langle A u_{\tau}^{k-1}, u_{\tau}^{k-1}\right\rangle+\tau\left(m_{B}-m_{J}\|M\|^{2}-\varepsilon-\tau c_{E} c_{q}\right)\left\|u_{\tau}^{k}\right\|^{2} \\
& \quad+\frac{m_{A}}{2}\left\|u_{\tau}^{k}-u_{\tau}^{k-1}\right\|^{2} \leq C \tau\left(\tau \sum_{j=1}^{k-1}\left\|u_{\tau}^{j}\right\|^{2}+\left\|f_{\tau}^{k}\right\|_{V^{*}}+1\right) .
\end{aligned}
$$

We now choose $\varepsilon=\frac{m_{B}-m_{J}\|M\|^{2}}{2}$ and $\tau_{0}=\frac{\varepsilon}{c_{E} c_{q}}$. Then, for all $\tau \in\left(0, \tau_{0}\right)$, it follows

$$
\begin{gathered}
\frac{1}{2}\left\langle A u_{\tau}^{k}, u_{\tau}^{k}\right\rangle-\frac{1}{2}\left\langle A u_{\tau}^{k-1}, u_{\tau}^{k-1}\right\rangle+\frac{m_{A}}{2}\left\|u_{\tau}^{k}-u_{\tau}^{k-1}\right\|^{2} \\
\leq C \tau\left(\tau \sum_{j=1}^{k-1}\left\|u_{\tau}^{j}\right\|^{2}+\left\|f_{\tau}^{k}\right\|_{V^{*}}+1\right) .
\end{gathered}
$$


Summing the above inequalities for $k=1, \ldots, n$, where $1 \leq n \leq N$, and then applying $H(A)$, we get

$$
\frac{m_{A}}{2}\left\|u_{\tau}^{k}\right\|^{2}+\frac{m_{A}}{2} \sum_{k=1}^{n}\left\|u_{\tau}^{k}-u_{\tau}^{k-1}\right\|^{2} \leq C\left(\tau \sum_{k=1}^{n-1}\left\|u_{\tau}^{k}\right\|^{2}+1\right) .
$$

Now, we use the discrete version of the Gronwall inequality in Lemma 5, to verify estimates (6) and (7). The estimate (8) follows directly from (6) and $H(J)$ (ii).

Denote $v_{\tau}^{k}=\frac{u_{\tau}^{k}-u_{\tau}^{k-1}}{\tau}$ for $k=1, \ldots, N$. We take $v=-v_{\tau}^{k}$ in (4) to get

$$
\begin{aligned}
& m_{A}\left\|v_{\tau}^{k}\right\|^{2}-\|B\|\left\|u_{\tau}^{k}\right\|\left\|v_{\tau}^{k}\right\|-\left\|\xi_{\tau}^{k}\right\|_{X^{*}}\|M\|\left\|v_{\tau}^{k}\right\|-\left\|x_{\tau}^{k}\right\|_{V^{*}}\left\|v_{\tau}^{k}\right\| \\
& \leq\left\langle\frac{A u_{\tau}^{k}-A u_{\tau}^{k-1}}{\tau}, v_{\tau}^{k}\right\rangle+\left\langle x_{\tau}^{k}, v_{\tau}^{k}\right\rangle+\left\langle B u_{\tau}^{k}, v_{\tau}^{k}\right\rangle+\left\langle\xi_{\tau}^{k}, M v_{\tau}^{k}\right\rangle_{X^{*} \times X} \\
& \quad \leq\left\langle f_{\tau}^{k}, v_{\tau}^{k}\right\rangle \leq\left\|f_{\tau}^{k}\right\|_{V^{*}}\left\|v_{\tau}^{k}\right\|,
\end{aligned}
$$

hence,

$$
m_{A}\left\|v_{\tau}^{k}\right\|^{2} \leq\left(\|B\|\left\|u_{\tau}^{k}\right\|+\left\|\xi_{\tau}^{k}\right\|_{X^{*}}\|M\|+\left\|x_{\tau}^{k}\right\|_{V^{*}}+\left\|f_{\tau}^{k}\right\|_{V^{*}}\right)\left\|v_{\tau}^{k}\right\|
$$

The latter together with (6), (8), $H(E), H(q)$, and the Cauchy inequality with $\varepsilon>0$ implies

$$
\left(m_{A}-\varepsilon\right)\left\|v_{\tau}^{k}\right\|^{2} \leq C\left(1+\left\|f_{\tau}^{k}\right\|_{V^{*}}\right) .
$$

We choose now $\varepsilon=\frac{m_{A}}{2}$ to get

$$
\tau \sum_{k=1}^{N}\left\|v_{\tau}^{k}\right\|^{2} \leq C\left(1+\tau \sum_{k=1}^{N}\left\|f_{\tau}^{k}\right\|_{V^{*}}\right) \leq C
$$

So, we obtain the estimate (9), which completes the proof of this lemma.

Subsequently, for a given $\tau>0$, we define the piecewise affine function $u_{\tau}$ and the piecewise constant interpolant functions $\bar{u}_{\tau}, \xi_{\tau}, f_{\tau}$, and $w_{\tau}$ as follows

$$
\begin{aligned}
& u_{\tau}(t)=u_{\tau}^{k}+\frac{t-t_{k}}{\tau}\left(u_{\tau}^{k}-u_{\tau}^{k-1}\right) \text { for } t \in\left(t_{k-1}, t_{k}\right], \\
& \bar{u}_{\tau}(t)=\left\{\begin{array}{l}
u_{\tau}^{k}, t \in\left(t_{k-1}, t_{k}\right], \\
u_{\tau}^{0}, t=0,
\end{array}\right. \\
& \xi_{\tau}(t)=\xi_{\tau}^{k}, \quad t \in\left(t_{k-1}, t_{k}\right], \\
& f_{\tau}(t)= \begin{cases}f_{\tau}^{k}, & t \in\left(t_{k-1}, t_{k}\right], \\
f(0), & t=0,\end{cases} \\
& w_{\tau}(t)= \begin{cases}\alpha+\sum_{j=1}^{k} \int_{t_{j-1}}^{t_{j}} q\left(t_{k}, s\right) u_{\tau}^{j} d s, t \in\left(t_{k-1}, t_{k}\right], \\
\alpha, & t=0 .\end{cases}
\end{aligned}
$$


Now, we rewrite Problem 8 in the following equivalent form

$$
\left\langle A u_{\tau}^{\prime}(t)+B \bar{u}_{\tau}(t)+E\left(w_{\tau}(t)\right), v\right\rangle+\left\langle\xi_{\tau}(t), M v\right\rangle_{X^{*} \times X}=\left\langle f_{\tau}(t), v\right\rangle
$$

for all $v \in V$ and a.e. $t \in(0, T)$, where $\xi_{\tau}(t) \in \partial J\left(M \bar{u}_{\tau}(t)\right)$ for a.e. $t \in(0, T)$.

The main results of this section are delivered in the following theorem.

Theorem 11 Under assumptions of Lemma 9, Problem 6 has a unique solution $u \in$ $H^{1}(0, T ; V)$.

Proof The bound (6) ensures that $\left\{\bar{u}_{\tau}\right\}$ is bounded in $\mathcal{V}$ due to the following inequality

$$
\left\|\bar{u}_{\tau}\right\|_{\mathcal{V}}^{2}=\tau \sum_{n=1}^{N}\left\|u_{\tau}^{n}\right\|^{2} \leq C .
$$

It follows from the reflexivity of $\mathcal{V}$ that there exists a function $u \in \mathcal{V}$ such that, passing to a subsequence again indexed by $\tau$, we have

$$
\bar{u}_{\tau} \rightarrow u \text { weakly in } \mathcal{V} \text {, as } \tau \rightarrow 0 \text {. }
$$

Also, from (6), we have that the sequence $\left\{u_{\tau}\right\}$ is bounded in $\mathcal{V}$, and therefore, there exists $u_{1} \in \mathcal{V}$ such that

$$
u_{\tau} \rightarrow u_{1} \text { weakly in } \mathcal{V}, \text { as } \tau \rightarrow 0 .
$$

Hence, we get $\bar{u}_{\tau}-u_{\tau} \rightarrow u-u_{1}$ weakly in $\mathcal{V}$, as $\tau \rightarrow 0$. By the Hölder inequality and the boundedness of $\left\{u_{\tau}^{\prime}\right\}$ (see (9))

$$
\left\|u_{\tau}^{\prime}\right\|_{\mathcal{V}}^{2}=\tau \sum_{k=1}^{N}\left\|v_{\tau}^{k}\right\|^{2} \leq C,
$$

we have

$$
\begin{aligned}
& \left\|\bar{u}_{\tau}-u_{\tau}\right\|_{\mathcal{V}}^{2}=\sum_{k=1}^{N} \int_{t_{k-1}}^{t_{k}}\left(t_{k}-s\right)^{2}\left\|v_{\tau}^{k}\right\|^{2} d s \\
& =\sum_{k=1}^{N} \int_{t_{k-1}}^{t_{k}}\left(t_{k}-s\right)^{2}\left\|u_{\tau}^{\prime}(s)\right\|^{2} d s \leq \frac{\tau^{2}}{3}\left\|u_{\tau}^{\prime}\right\|_{\mathcal{V}}^{2} .
\end{aligned}
$$

From estimate (15), we deduce that $u=u_{1}$. On the other hand, by the boundedness of $\left\{u_{\tau}^{\prime}\right\}$ (see (9)), we also obtain (cf. [42, Proposition 23.19, p. 419])

$$
u_{\tau}^{\prime} \rightarrow u^{\prime} \text { weakly in } \mathcal{V} \text {, as } \tau \rightarrow 0 .
$$

In addition, using the boundedness of $\left\{\xi_{\tau}\right\}$ (see (8)) and the reflexivity of the space $\mathcal{X}^{*}$, we conclude

$$
\xi_{\tau} \rightarrow \xi \text { weakly in } \mathcal{X}^{*} \text {, as } \tau \rightarrow 0 \text { with } \xi \in \mathcal{X}^{*} \text {. }
$$


By virtue of the hypothesis $H(q)$ and boundedness of $\left\{\bar{u}_{\tau}\right\}$ (see (6)), one has the following estimate for $t \in\left(t_{k-1}, t_{k}\right]$

$$
\begin{aligned}
& \left\|\int_{0}^{t} q(t, s) \bar{u}_{\tau}(s) d s-\int_{0}^{t_{k}} q\left(t_{k}, s\right) \bar{u}_{\tau}(s) d s\right\| \leq \int_{t}^{t_{k}}\left\|q\left(t_{k}, s\right) \bar{u}_{\tau}(s)\right\| d s \\
& \quad+\int_{0}^{t}\left\|\left(q(t, s)-q\left(t_{k}, s\right)\right) \bar{u}_{\tau}(s)\right\| d s \leq C_{0} \tau
\end{aligned}
$$

for some $C_{0}>0$, which is independent of $\tau$. Moreover, [4, Lemma 3.3] implies that

$$
f_{\tau} \rightarrow f \text { strongly in } \mathcal{V}^{*}, \quad \text { as } \tau \rightarrow 0 \text {. }
$$

Next, we shall show that $u$ is a solution of Problem 6. To this end, we define the Nemytskii operators $\mathcal{A}, \mathcal{B}: \mathcal{V} \rightarrow \mathcal{V}^{*}$ by $(\mathcal{A} v)(t)=A(v(t))$ and $(\mathcal{B} v)(t)=B(v(t))$ for all $v \in \mathcal{V}$ and a.e. $t \in(0, T)$. From hypotheses $H(A)$ and $H(B)$, it is clear that $\mathcal{A}$ and $\mathcal{B}$ are both linear and bounded, so they are also weakly continuous. Thus, from (16) and (13), we obtain $\mathcal{A} u_{\tau}^{\prime} \rightarrow \mathcal{A} u^{\prime}$ and $\mathcal{B} \bar{u}_{\tau} \rightarrow \mathcal{B} u$ both weakly in $\mathcal{V}^{*}$, as $\tau \rightarrow 0$, i.e.,

$$
\lim _{\tau \rightarrow 0}\left\langle\mathcal{A} u_{\tau}^{\prime}, v\right\rangle_{\mathcal{V}^{*} \times \mathcal{V}}=\left\langle\mathcal{A} u^{\prime}, v\right\rangle_{\mathcal{V}^{*} \times \mathcal{V}} \text { and } \lim _{\tau \rightarrow 0}\left\langle\mathcal{B} \bar{u}_{\tau}, v\right\rangle_{\mathcal{V}^{*} \times \mathcal{V}}=\langle\mathcal{B} u, v\rangle_{\mathcal{V}^{*} \times \mathcal{V}}
$$

for all $v \in \mathcal{V}$. Now, we consider the Nemitskii operators $\mathcal{E}, \mathcal{E}_{2}: \mathcal{V} \rightarrow \mathcal{V}^{*}$ by

$$
(\mathcal{E} v)(t)=E\left(\int_{0}^{t} q(t, s) v(s) d s\right) \text { and }\left(\mathcal{E}_{2} v\right)(t)=E v(t)
$$

for all $v \in \mathcal{V}$ and a.e. $t \in(0, T)$. It is obvious that $\mathcal{E}$ is weakly continuous being bounded and linear. From the convergence (13), one has

$$
\lim _{\tau \rightarrow 0}\left\langle\mathcal{E} \bar{u}_{\tau}, v\right\rangle_{\mathcal{V}^{*} \times \mathcal{V}}=\langle\mathcal{E} u, v\rangle_{\mathcal{V}^{*} \times \mathcal{V}}
$$

for all $v \in \mathcal{V}$. Next, from $H(E), H(q)$ and (18), we have

$$
\mathcal{E}_{2}\left(w_{\tau}-\alpha\right)-\mathcal{E}\left(\bar{u}_{\tau}\right) \rightarrow 0 \text { strongly in } \mathcal{V}^{*}, \quad \text { as } \tau \rightarrow 0
$$

which implies

$$
\begin{aligned}
& \lim _{\tau \rightarrow 0}\left\langle\mathcal{E}_{2}\left(w_{\tau}\right), v\right\rangle_{\mathcal{V}^{*} \times V} \\
& =\lim _{\tau \rightarrow 0}\left(\left\langle\mathcal{E}_{2}\left(w_{\tau}-\alpha\right)-\mathcal{E}\left(\bar{u}_{\tau}\right), v\right\rangle_{\mathcal{V}^{*} \times \mathcal{V}}+\left\langle\mathcal{E}\left(\bar{u}_{\tau}\right), v\right\rangle_{\mathcal{V}^{*} \times \mathcal{V}}+\left\langle\mathcal{E}_{2}(\alpha), v\right\rangle_{\mathcal{V}^{*} \times \mathcal{V}}\right) \\
& \quad=\langle\mathcal{E} u, v\rangle_{\mathcal{V}^{*} \times \mathcal{V}}+\left\langle\mathcal{E}_{2}(\alpha), v\right\rangle_{\mathcal{V}^{*} \times \mathcal{V}}
\end{aligned}
$$

for all $v \in \mathcal{V}$.

Since the embedding $H^{1}(0, T ; V) \subset C(0, T ; V)$ is continuous, from the convergences (14) and (16), by [24, Lemma 4(a)], we have

$$
u_{\tau}(t) \rightarrow u(t) \text { weakly in } V, \quad \text { as } \tau \rightarrow 0,
$$

for all $t \in[0, T]$. Using the convergence $\bar{u}_{\tau}-u_{\tau} \rightarrow 0$ strongly in $\mathcal{V}$, as $\tau \rightarrow 0$, by the converse Lebesgue dominated convergence theorem ([23, Theorem 2.39]), we may assume that $\bar{u}_{\tau}(t)-u_{\tau}(t) \rightarrow 0$ strongly in $V$ for a.e. $t \in(0, T)$, as $\tau \rightarrow 0$. This together with (22) implies

$$
\bar{u}_{\tau}(t) \rightarrow u(t) \text { weakly in } V, \text { for a.e. } t \in(0, T) .
$$


From the compactness of the operator $M$, we deduce $M \bar{u}_{\tau}(t) \rightarrow M u(t)$ strongly in $X$ for a.e. $t \in(0, T)$. Since $\xi_{\tau}(t) \in \partial J\left(M \bar{u}_{\tau}(t)\right)$ for a.e. $t \in(0, T)$, we use also the convergence (17), and by [2, Theorem 1, Section 1.4], we have

$$
\xi(t) \in \partial J(M u(t)) \text { for a.e. } t \in(0, T) .
$$

Now, we introduce the Nemitskii operator $\mathcal{M}: \mathcal{V} \rightarrow \mathcal{X}$ defined by $(\mathcal{M} v)(t)=$ $M(v(t))$ for all $v \in \mathcal{V}$ and a.e. $t \in(0, T)$, so, from (17), we have

$$
\lim _{\tau \rightarrow 0}\left\langle\xi_{\tau}, \mathcal{M} v\right\rangle_{\mathcal{X} * \times \mathcal{X}}=\langle\xi, \mathcal{M} v\rangle_{\mathcal{X}^{*} \times \mathcal{X}}
$$

for all $v \in \mathcal{V}$.

From (19)-(21), (23), and (24), we infer that

$$
\left\langle\mathcal{A} u^{\prime}+\mathcal{B} u+\mathcal{E} u+\mathcal{E}_{2} \alpha, v\right\rangle_{\mathcal{V}^{*} \times \mathcal{V}}+\langle\xi, \mathcal{M} v\rangle_{\mathcal{X}^{*} \times \mathcal{X}}=\langle f, v\rangle_{\mathcal{V}^{*} \times \mathcal{V}}
$$

for all $v \in \mathcal{V}$ with $\xi(t) \in \partial J(M u(t))$ for a.e. $t \in(0, T)$. Furthermore, we shall show that $u \in \mathcal{V}$ with $u^{\prime} \in \mathcal{V}$ is also a solution of Problem 6. Arguing by contradiction, we suppose that $u$ is not a solution to Problem 6. This means there exists a measurable set $I \subset[0, T]$ with meas(I) $>0$ and $v^{*} \in V$ such that

$$
\left\langle A u^{\prime}(t)+B u(t)+(\mathcal{R} u)(t), v^{*}\right\rangle+J^{0}\left(M u(t) ; M v^{*}\right)<\left\langle f(t), v^{*}\right\rangle \text { for a.e. } t \in I .
$$

We now denote a function $\tilde{v} \in \mathcal{V}$ by

$$
\widetilde{v}(t)= \begin{cases}v^{*} & \text { if } t \in I \\ 0 & \text { otherwise. }\end{cases}
$$

Inserting $v=\widetilde{v}$ into (25) and taking account of (26), it follows from [23, Theorem 3.47] that

$$
\begin{aligned}
& \int_{I}\left\langle f(t), v^{*}\right\rangle d t \leq \int_{I}\left\langle A u^{\prime}(t)+B u(t)+(\mathcal{R} u)(t)-f(t), v^{*}\right\rangle+J^{0}\left(M u(t) ; M v^{*}\right) d t \\
& \quad<\int_{I}\left\langle f(t), v^{*}\right\rangle d t .
\end{aligned}
$$

This results a contradiction, so, $u \in \mathcal{V}$ with $u^{\prime} \in \mathcal{V}$ is also a solution of Problem 6 .

Finally, we will verify that the solution of Problem 6 is unique. Let $u_{1}$ and $u_{2}$ be two solutions of Problem 6. Then,

$$
\left\langle A u_{1}^{\prime}(t)+B u_{1}(t)+\left(\mathcal{R} u_{1}\right)(t)-f(t), v\right\rangle+J^{0}\left(M u_{1}(t) ; M v\right) \geq 0
$$

and

$$
\left\langle A u_{2}^{\prime}(t)+B u_{2}(t)+\left(\mathcal{R} u_{2}\right)(t)-f(t), v\right\rangle+J^{0}\left(M u_{2}(t) ; M v\right) \geq 0
$$

for all $v \in V$ and a.e. $t \in(0, T)$. Taking $v=u_{2}(t)-u_{1}(t)$ in the first inequality and $v=u_{1}(t)-u_{2}(t)$ in the second one, we add the resulting inequalities to get

$$
\begin{aligned}
& \left\langle A u_{1}^{\prime}(t)-A u_{2}^{\prime}(t), u_{1}(t)-u_{2}(t)\right\rangle+\left\langle B u_{1}(t)-B u_{2}(t), u_{1}(t)-u_{2}(t)\right\rangle \\
& \quad \leq J^{0}\left(M u_{1}(t) ; M v\right)+J^{0}\left(M u_{1}(t) ; M u\right)+\left\langle\left(\mathcal{R} u_{1}\right)(t)-\left(\mathcal{R} u_{2}\right)(t), u_{2}(t)-u_{1}(t)\right\rangle
\end{aligned}
$$


for a.e. $t \in(0, T)$. We use the assumptions $H(A), H(B), H(E), H(q)$, and $H(J)($ iii) to obtain

$$
\begin{aligned}
& \frac{1}{2} \frac{d}{d t}\left\langle A\left(u_{1}(t)-u_{2}(t)\right), u_{1}(t)-u_{2}(t)\right\rangle+\left(m_{B}-m_{J}\|M\|^{2}\right)\left\|u_{1}(t)-u_{2}(t)\right\|^{2} \\
& \quad \leq c_{E} c_{q} \int_{0}^{t}\left\|u_{1}(s)-u_{2}(s)\right\|\left\|u_{1}(t)-u_{2}(t)\right\| d s .
\end{aligned}
$$

We integrate this inequality on $[0, t]$, where $t \in[0, T]$, and use $H(A)$ (ii) and $\left(H_{0}\right)$ to deduce

$$
\begin{aligned}
& \frac{m_{A}}{2}\left\|u_{1}(t)-u_{2}(t)\right\|^{2} \leq \frac{1}{2}\left\langle A\left(u_{1}(t)-u_{2}(t)\right), u_{1}(t)-u_{2}(t)\right\rangle \\
& \quad \leq c_{E} c_{q} \int_{0}^{t}\left\|u_{1}(s)-u_{2}(s)\right\| \int_{0}^{s}\left\|u_{1}(\eta)-u_{2}(\eta)\right\| d \eta d s \\
& \quad \leq c_{E} c_{q}\left(\int_{0}^{t}\left\|u_{1}(s)-u_{2}(s)\right\| d s\right)^{2}
\end{aligned}
$$

for all $t \in[0, T]$. Hence,

$$
\left\|u_{1}(t)-u_{2}(t)\right\| \leq\left(\frac{2 c_{E} c_{q}}{m_{A}}\right)^{\frac{1}{2}} \int_{0}^{t}\left\|u_{1}(s)-u_{2}(s)\right\| d s
$$

for all $t \in[0, T]$. Finally, we use the Gronwall inequality (see, e.g., [36, Lemma 2.31]) to obtain $u_{1}=u_{2}$. This completes the proof of the theorem.

\section{A fully discrete approximation scheme}

In this section, we study a fully discrete approximation scheme for the historydependent hemivariational inequality stated in Problem 6. In this method, the time variable is discretized by finite difference and the spatial variable is approximated by finite elements.

Assume that $V^{h}$ is a finite dimensional subspace of $V$ and $u_{0}^{h} \in V^{h}$ is an approximation of the initial point $u_{0} \in V$. For $N \in \mathbb{N}, N>0$ given, we denote the time step length by $k=\frac{T}{N}$ and $t_{n}=k n$ for $n=0, \ldots, N$. For a continuous function $g$ defined on the interval $[0, T]$, in the sequel, we will write $g_{n}=g\left(t_{n}\right)$ for $n=0, \ldots, N$. In addition, for a sequence $\left\{u_{n}\right\}_{n=0}^{N}$, we use the notation

$$
\delta u_{n}=\frac{u_{n}-u_{n-1}}{k}, n=1, \ldots, N .
$$

For the history-dependent operator

$$
(\mathcal{R} v)(t)=\int_{0}^{t} q(t, s) v(s) d s \text { for } v \in C(0, T ; V), t \in[0, T],
$$

we introduce a modified trapezoidal approximation for $\mathcal{R}$ defined by

$$
\mathcal{R}_{n}^{k} v=E\left(\sum_{j=1}^{n} \int_{t_{j-1}}^{t_{j}} q\left(t_{n}, s\right) v_{j} d s+\alpha\right)
$$


for $v=\left\{v_{j}\right\}_{j=1}^{N}$. In addition, if $w \in C(0, T ; V)$, then the expression $\mathcal{R}_{n}^{k} w$ is understood as follows

$$
\mathcal{R}_{n}^{k} w=E\left(\sum_{j=1}^{n} \int_{t_{j-1}}^{t_{j}} q\left(t_{n}, s\right) w\left(t_{j}\right) d s+\alpha\right) .
$$

Subsequently, we consider the following fully discrete approximation problem for Problem 6.

Problem 12 Find $u^{h k}=\left\{u_{n}^{h k}\right\} \subset V^{h}$ such that $u_{0}^{h k}=u_{0}^{h}$ and

$$
\begin{gathered}
\left\langle A \delta u_{n}^{h k}+B u_{n}^{h k}+\mathcal{R}_{n}^{k} u^{h k}, v^{h}-u_{n}^{h k}\right\rangle+J^{0}\left(M u_{n}^{h k} ; M v^{h}-M u_{n}^{h k}\right) \\
\geq\left\langle f_{n}, v^{h}-u_{n}^{h k}\right\rangle \text { for all } v^{h} \in V^{h}
\end{gathered}
$$

for all $n=1,2, \ldots, N$.

We will provide an error analysis of the fully discrete approximation (28). Our goal is to prove the Céa-type inequality for Problem 12.

First, exploiting the definition of $\delta u_{n}^{h k}$, the inequality (28) can be reformulated as follows

$$
\begin{array}{r}
\left\langle A u_{n}^{h k}+k B u_{n}^{h k}+k \mathcal{R}_{n}^{k} u^{h k}, v^{h}-u_{n}^{h k}\right\rangle+k J^{0}\left(M u_{n}^{h k} ; M v^{h}-M u_{n}^{h k}\right) \\
\geq\left\langle k f_{n}+A u_{n-1}^{h k}, v^{h}-u_{n}^{h k}\right\rangle \text { for all } v^{h} \in V^{h} .
\end{array}
$$

This inequality represents a stationary hemivariational inequality. When $k$ is small enough, from Lemma 9, we know that under the hypotheses $H(A), H(B), H(E)$, $H(q), H(J), H(M)$, and $\left(H_{0}\right)$, it has a unique solution $u_{n}^{h k} \in V^{h}$. Moreover, Theorem 11 reveals that Problem 6 has a unique solution $u \in H^{1}(0, T ; V)$.

Since $A \in \mathcal{L}\left(V, V^{*}\right)$ is coercive, in what follows, for a convenience, we introduce the norm $\|\cdot\|_{A}$ by $\|v\|_{A}^{2}=\langle A v, v\rangle$ for all $v \in V$, which is equivalent to the norm $\|\cdot\|_{V}$. In the sequel, we denote by $C>0$ a constant which may differ from line to line, but it is independent of $h$ and $k$.

For an error analysis, we have from (1) at $t=t_{n}$ that

$$
\left\langle A u_{n}^{\prime}+B u_{n}+\mathcal{R}_{n} u, v-u_{n}\right\rangle+J^{0}\left(M u_{n} ; M v-M u_{n}\right) \geq\left\langle f_{n}, v-u_{n}\right\rangle
$$

for all $v \in V$, where $\mathcal{R}_{n} u=(\mathcal{R} u)\left(t_{n}\right)$. Denote the errors

$$
\delta_{n}=\delta u_{n}-u_{n}^{\prime} \quad \text { and } \quad e_{n}=u_{n}-u_{n}^{h k}
$$

for $n=1,2, \ldots, N$. Taking $v=u_{n}^{h k}$ in (30), one has

$$
\begin{gathered}
\left\langle A \delta u_{n}+B u_{n}+\mathcal{R}_{n} u, u_{n}^{h k}-u_{n}\right\rangle+J^{0}\left(M u_{n} ; M u_{n}^{h k}-M u_{n}\right) \\
\geq\left\langle f_{n}, u_{n}^{h k}-u_{n}\right\rangle+\left\langle A \delta_{n}, u_{n}^{h k}-u_{n}\right\rangle .
\end{gathered}
$$


We add (31) and (28) to get

$$
\begin{aligned}
& \left\langle A \delta u_{n}+B u_{n}+\mathcal{R}_{n} u, u_{n}^{h k}-u_{n}\right\rangle+\left\langle A \delta u_{n}^{h k}+B u_{n}^{h k}+\mathcal{R}_{n}^{k} u^{h k}, v^{h}-u_{n}^{h k}\right\rangle \\
& +J^{0}\left(M u_{n} ; M u_{n}^{h k}-M u_{n}\right)+J^{0}\left(M u_{n}^{h k} ; M v^{h}-M u_{n}^{h k}\right) \\
& \quad \geq\left\langle f_{n}, v^{h}-u_{n}\right\rangle+\left\langle A \delta_{n}, u_{n}^{h k}-u_{n}\right\rangle
\end{aligned}
$$

for all $v^{h} \in V^{h}$. Hence,

$$
\begin{aligned}
& \left\langle A \delta\left(u_{n}-u_{n}^{h k}\right)+B\left(u_{n}-u_{n}^{h k}\right), u_{n}^{h k}-u_{n}\right\rangle+\left\langle\mathcal{R}_{n} u-\mathcal{R}_{n}^{k} u^{h k}, u_{n}^{h k}-u_{n}\right\rangle \\
& +\left\langle A \delta u_{n}^{h k}+B u_{n}^{h k}+\mathcal{R}_{n}^{k} u^{h k}, v^{h}-u_{n}\right\rangle+J^{0}\left(M u_{n} ; M u_{n}^{h k}-M u_{n}\right) \\
& +J^{0}\left(M u_{n}^{h k} ; M v^{h}-M u_{n}^{h k}\right) \geq\left\langle f_{n}, v^{h}-u_{n}\right\rangle+\left\langle A \delta_{n}, u_{n}^{h k}-u_{n}\right\rangle
\end{aligned}
$$

for all $v^{h} \in V^{h}$. We use the fact that the function $v \mapsto J^{0}(M u ; M v)$ is subadditive (see, e.g., [23, Proposition 3.23(i)]), to obtain

$$
J^{0}\left(M u_{n}^{h k} ; M v^{h}-M u_{n}^{h k}\right) \leq J^{0}\left(M u_{n}^{h k} ; M v^{h}-M u_{n}\right)+J^{0}\left(M u_{n}^{h k} ; M u_{n}-M u_{n}^{h k}\right) .
$$

So, we have

$$
\begin{aligned}
& \left\langle A \delta\left(u_{n}-u_{n}^{h k}\right)+B\left(u_{n}-u_{n}^{h k}\right), u_{n}^{h k}-u_{n}\right\rangle+\left\langle\mathcal{R}_{n} u-\mathcal{R}_{n}^{k} u^{h k}, u_{n}^{h k}-u_{n}\right\rangle \\
& +\left\langle A \delta u_{n}^{h k}+B u_{n}^{h k}+\mathcal{R}_{n}^{k} u^{h k}, v^{h}-u_{n}\right\rangle+J^{0}\left(M u_{n} ; M u_{n}^{h k}-M u_{n}\right) \\
& +J^{0}\left(M u_{n}^{h k} ; M v^{h}-M u_{n}\right)+J^{0}\left(M u_{n}^{h k} ; M u_{n}-M u_{n}^{h k}\right) \\
& \quad \geq\left\langle f_{n}, v^{h}-u_{n}\right\rangle+\left\langle A \delta_{n}, u_{n}^{h k}-u_{n}\right\rangle
\end{aligned}
$$

for all $v^{h} \in V^{h}$. Combining this inequality with the identity

$$
\langle A(u-v), u\rangle=\frac{1}{2}(\langle A u, u\rangle-\langle A v, v\rangle+\langle A(u-v), u-v\rangle)
$$

and using the hypotheses $H(B)$ and $H(J)($ iii) (see Remark 7), it follows that

$$
\begin{aligned}
& \frac{1}{2 k}\left(\left\|e_{n}\right\|_{A}^{2}-\left\|e_{n-1}\right\|_{A}^{2}\right)+m_{B}\left\|e_{n}\right\|^{2}-m_{J}\|M\|^{2}\left\|e_{n}\right\|^{2}-\left\|\mathcal{R}_{n} u-\mathcal{R}_{n}^{k} u^{h k}\right\| V^{*}\left\|e_{n}\right\| \\
& \leq\left\langle A \delta u_{n}^{h k}+B u_{n}^{h k}+\mathcal{R}_{n}^{k} u^{h k}-f_{n}, v^{h}-u_{n}\right\rangle+J^{0}\left(M u_{n}^{h k} ; M v^{h}-M u_{n}\right) \\
& \quad-\left\langle A \delta_{n}, u_{n}^{h k}-u_{n}\right\rangle
\end{aligned}
$$

for all $v^{h} \in V^{h}$. Furthermore, we introduce a residual-type quantity by

$$
S_{n}(v)=\left\langle A u_{n}^{\prime}+B u_{n}+\mathcal{R}_{n} u-f_{n}, v-u_{n}\right\rangle+J^{0}\left(M u_{n} ; M v-M u_{n}\right) \text { for } v \in V .
$$

Using the fact that $u \in H^{1}(0, T ; V)$ (see Theorem 11), we have

$$
\begin{aligned}
& \left\|\mathcal{R}_{n} u-\mathcal{R}_{n}^{k} u\right\|_{V^{*}} \leq c_{E} \sum_{i=1}^{n} \int_{t_{i-1}}^{t_{i}}\left\|q\left(t_{n}, s\right)\left(u(s)-u_{i}\right)\right\| d s \\
& \quad \leq C_{1} k \sum_{i=1}^{n} \int_{t_{i-1}}^{t_{i}}\left\|u^{\prime}(s)\right\| d s=C_{1} k \int_{0}^{T}\left\|u^{\prime}(s)\right\| d s \leq C_{1} \sqrt{T}\left\|u^{\prime}\right\|_{\mathcal{V}} k
\end{aligned}
$$


with some $C_{1}>0$, and

$$
\left\|\mathcal{R}_{n}^{k} u-\mathcal{R}_{n}^{k} u^{h k}\right\|_{V^{*}} \leq c_{E} c_{q} \sum_{i=1}^{n} \int_{t_{i-1}}^{t_{i}}\left\|u_{i}-u_{i}^{h k}\right\| d s \leq k c_{E} c_{q} \sum_{i=1}^{n}\left\|u_{i}-u_{i}^{h k}\right\| .
$$

From these inequalities, we obtain

$$
\left\|\mathcal{R}_{n} u-\mathcal{R}_{n}^{k} u^{h k}\right\|_{V^{*}} \leq\left\|\mathcal{R}_{n} u-\mathcal{R}_{n}^{k} u\right\|_{V^{*}}+\left\|\mathcal{R}_{n}^{k} u-\mathcal{R}_{n}^{k} u^{h k}\right\|_{V^{*}} \leq C_{2} k\left(1+\sum_{j=1}^{n}\left\|e_{j}\right\|\right),
$$

where $C_{2}=\max \left\{c_{E} c_{q}, C_{1} \sqrt{T}\left\|u^{\prime}\right\|_{\mathcal{V}}\right\}$. Therefore, from (32), we have

$$
\begin{aligned}
& \frac{1}{2 k}\left(\left\|e_{n}\right\|_{A}^{2}-\left\|e_{n-1}\right\|_{A}^{2}\right)-C_{2} k\left(1+\sum_{j=1}^{n}\left\|e_{j}\right\|\right)\left\|e_{n}\right\|+\left(m_{B}-m_{J}\|M\|^{2}\right)\left\|e_{n}\right\|^{2} \\
& \leq \frac{1}{k}\left\langle A e_{n}-A e_{n-1}, u_{n}-v_{n}^{h}\right\rangle+\left\langle B e_{n}, u_{n}-v_{n}^{h}\right\rangle+\left\langle\mathcal{R}_{n} u-\mathcal{R}_{n}^{k} u^{h k}, u_{n}-v_{n}^{h}\right\rangle \\
&+\left\langle\xi_{n}-\xi_{n}^{h k}, M\left(u_{n}-v_{n}^{h}\right)\right\rangle_{X^{*} \times X}+S_{n}\left(v_{n}^{h}\right)+\left\langle A \delta_{n}, v_{n}^{h}-u_{n}\right\rangle+\left\langle A \delta_{n}, e_{n}\right\rangle
\end{aligned}
$$

where $\xi_{n} \in \partial J\left(M u_{n}\right)$ and $\xi_{n}^{h k} \in \partial J\left(M u_{n}^{k h}\right)$.

Note that the hypothesis $H(J)$ (ii) and $u \in H^{1}(0, T ; V)$ imply that the sequence $\left\{\left\|\xi_{n}\right\|_{X^{*}}\right\}$ is uniformly bounded. It follows from Lemma 10 that $\left\{\left\|\xi_{n}^{h k}\right\|_{X^{*}}\right\}$ is uniformly bounded as well. Hence, we have

$$
\left\langle\xi_{n}-\xi_{n}^{h k}, M\left(u_{n}-v_{n}^{h}\right)\right\rangle_{X^{*} \times X} \leq C\left\|M\left(u_{n}-v_{n}^{h}\right)\right\|_{X} .
$$

Applying (33) again, we obtain

$$
\left\langle\mathcal{R}_{n} u-\mathcal{R}_{n}^{k} u_{n}^{h k}, u_{n}-v_{n}^{h}\right\rangle \leq C_{2} k\left(1+\sum_{j=1}^{n}\left\|e_{j}\right\|\right)\left\|u_{n}-v_{n}^{h}\right\|
$$

Combining (34)-(36) and applying the Cauchy inequality with $\varepsilon>0$, we have

$$
\begin{aligned}
& \left\|e_{n}\right\|_{A}^{2}-\left\|e_{n-1}\right\|_{A}^{2}+2 k\left(m_{B}-m_{J}\|M\|^{2}\right)\left\|e_{n}\right\|^{2} \leq 2\left\langle A e_{n}-A e_{n-1}, u_{n}-v_{n}^{h}\right\rangle \\
& +C k\left\|u_{n}-v_{n}^{h}\right\|^{2}+\varepsilon k\left\|e_{n}\right\|^{2}+C k^{2} \sum_{j=1}^{n-1}\left\|e_{j}\right\|^{2}+C k^{3}+C_{2} k^{2}\left\|e_{n}\right\|^{2} \\
& \quad+C k\left\|M\left(u_{n}-v_{n}^{h}\right)\right\|_{X}+2 k\left|S_{n}\left(v_{n}^{h}\right)\right|+C k\left\|A \delta_{n}\right\|_{V^{*}}^{2} .
\end{aligned}
$$

Now, we take $\varepsilon=m_{B}-m_{J}\|M\|^{2}$ and $k_{0}=\frac{m_{B}-m_{J}\|M\|^{2}}{C_{2}}$, which implies

$$
2\left(m_{B}-m_{J}\|M\|^{2}\right)-\varepsilon-k C_{2}>0,
$$

for all $k<k_{0}$. Subsequently, from (37), we have

$$
\begin{gathered}
\left\|e_{n}\right\|_{A}^{2}-\left\|e_{n-1}\right\|_{A}^{2} \leq 2\left\langle A e_{n}-A e_{n-1}, u_{n}-v_{n}^{h}\right\rangle+C k\left\|u_{n}-v_{n}^{h}\right\|^{2}+C k^{3} \\
+C k^{2} \sum_{j=1}^{n-1}\left\|e_{j}\right\|^{2}+C k\left\|M\left(u_{n}-v_{n}^{h}\right)\right\|_{X}+2 k\left|S_{n}\left(v_{n}^{h}\right)\right|+C k\left\|A \delta_{n}\right\|_{V^{*}}^{2} .
\end{gathered}
$$


Now, we replace $n$ by $l$ in the above inequality, and then sum it from 1 to $n$, where $1 \leq n \leq N$ to get

$$
\begin{aligned}
& \left\|e_{n}\right\|_{A}^{2} \leq\left\|e_{0}\right\|_{A}^{2}+2\left\langle A e_{n}, u_{n}-v_{n}^{h}\right\rangle+2 \sum_{l=1}^{n-1}\left\langle A e_{l},\left(u_{l}-v_{l}^{h}\right)-\left(u_{l+1}-v_{l+1}^{h}\right)\right\rangle \\
& +C k \sum_{l=1}^{n}\left(\left\|u_{l}-v_{l}^{h}\right\|^{2}+\left\|M\left(u_{l}-v_{l}^{h}\right)\right\|_{X}+\left|S_{l}\left(v_{l}^{h}\right)\right|+\left\|A \delta_{l}\right\|_{V^{*}}^{2}\right) \\
& -2\left\langle A e_{0}, u_{1}-v_{1}^{h}\right\rangle+C k^{2}+C k \sum_{l=1}^{n-1}\left\|e_{l}\right\|^{2} .
\end{aligned}
$$

This together with the following estimates

$$
\begin{aligned}
& 2\left\langle A e_{n}, u_{n}-v_{n}^{h}\right\rangle \leq \frac{1}{2}\left\|e_{n}\right\|_{A}^{2}+C\left\|u_{n}-v_{n}^{h}\right\|^{2}, \\
& -2\left\langle A e_{0}, u_{1}-v_{1}^{h}\right\rangle \leq\left\|e_{0}\right\|_{A}^{2}+C\left\|u_{1}-v_{1}^{h}\right\|^{2}
\end{aligned}
$$

and

$$
\begin{aligned}
& 2 \sum_{l=1}^{n-1}\left\langle A e_{l},\left(u_{l}-v_{l}^{h}\right)-\left(u_{l+1}-v_{l+1}^{h}\right)\right\rangle \leq 2 k\|A\| \sum_{l=1}^{n-1}\left\|e_{l}\right\|\left\|\delta\left(u_{l+1}-v_{l+1}^{h}\right)\right\| \\
& \quad \leq C k\left(\sum_{l=1}^{n-1}\left\|e_{l}\right\|^{2}+\sum_{l=2}^{n}\left\|\delta\left(u_{l}-v_{l}^{h}\right)\right\|^{2}\right)
\end{aligned}
$$

implies that

$$
\begin{aligned}
& \frac{1}{2}\left\|e_{n}\right\|_{A}^{2} \leq 2\left\|e_{0}\right\|_{A}^{2}+C\left\|u_{1}-v_{1}^{h}\right\|^{2}+C\left\|u_{n}-v_{n}^{h}\right\|^{2}+C k \sum_{l=1}^{n-1}\left\|e_{l}\right\|^{2}+C k^{2} \\
& +C k \sum_{l=1}^{n}\left(\left\|\delta\left(u_{l}-v_{l}^{h}\right)\right\|^{2}+\left\|u_{l}-v_{l}^{h}\right\|^{2}+\left\|M\left(u_{l}-v_{l}^{h}\right)\right\|_{X}+\left|S_{l}\left(v_{l}^{h}\right)\right|+\left\|A \delta_{l}\right\|_{V^{*}}^{2}\right) .
\end{aligned}
$$

It follows from the discrete Gronwall inequality, $H(A)$, and Lemma 5, that

$$
\begin{array}{r}
\max _{0 \leq n \leq N}\left\|e_{n}\right\|^{2} \leq C\left[k \sum_{l=1}^{N}\left(\left\|\delta\left(u_{l}-v_{l}^{h}\right)\right\|^{2}+\left\|M\left(u_{l}-v_{l}^{h}\right)\right\| X+\left|S_{l}\left(v_{l}^{h}\right)\right|+\left\|\delta_{l}\right\|^{2}\right)\right. \\
\left.+\left\|e_{0}\right\|^{2}+k^{2}+\max _{0 \leq n \leq N}\left\|u_{n}-v_{n}^{h}\right\|^{2}\right]
\end{array}
$$

for all $v_{n}^{h} \in V^{h}$.

We now summarize the results of the section in the form of a theorem. 
Theorem 13 Suppose that assumptions of Lemma 9 are satisfied. Let $u^{h k} \in V^{h}$ and $u \in H^{1}(0, T ; V)$ be the solutions of Problems 12 and 6 , respectively. Then, we have the estimate

$$
\begin{gathered}
\max _{0 \leq n \leq N}\left\|u_{n}-u_{n}^{h k}\right\|^{2} \leq C\left[k \sum_{l=1}^{N}\left(\left\|\delta\left(u_{l}-v_{l}^{h}\right)\right\|^{2}+\left\|M\left(u_{l}-v_{l}^{h}\right)\right\|_{X}+\left|S_{l}\left(v_{l}^{h}\right)\right|+\left\|\delta_{l}\right\|^{2}\right)\right. \\
\left.+\left\|e_{0}\right\|^{2}+k^{2}+\max _{0 \leq n \leq N}\left\|u_{n}-v_{n}^{h}\right\|^{2}\right]
\end{gathered}
$$

for all $v_{n}^{h} \in V^{h}$.

The inequality (39) is called the Céa-type inequality of the fully discrete approximation problem (Problem 12).

\section{A quasistatic viscoelastic contact problem}

In this section, we study the quasistatic contact problem between a viscoelastic body and a foundation. The volume forces and surface tractions are supposed to change slowly in time and therefore the acceleration in the system is negligible. Neglecting the inertial terms in the equation of motion leads to the quasistatic approximation for the process. We show that the variational formulation of the quasistatic contact problem is a time-dependent hemivariational inequality in Problem 6. For the latter, we apply the abstract result stated in Theorem 11 and prove a result on existence and uniqueness of weak solution. Further, we use the fully discrete approximation method discussed in Section 5 to study the numerical analysis of this contact problem and establish the result concerning optimal error estimate for the fully discrete scheme.

\subsection{Mathematical model and its variational formulation}

The physical setting of the contact problem is as follows. A deformable viscoelastic body occupies an open bounded subset $\Omega$ of $\mathbb{R}^{d}, d=2,3$ in applications. The volume forces of density $\boldsymbol{f}_{0}$ act in $\Omega$ and surface tractions of density $\boldsymbol{f}_{N}$ are applied on $\Gamma_{2}$. They both can depend on time. We are interested in the quasistatic process of the mechanical state of the body on the time interval $[0, T]$ with $0<T<+\infty$. The boundary $\Gamma=\partial \Omega$ of $\Omega$ is assumed to be Lipschitz continuous and it consists of three measurable parts $\Gamma_{1}, \Gamma_{2}$, and $\Gamma_{3}$ which are mutually disjoint, and $m\left(\Gamma_{1}\right)>0$. The unit outward normal vector $\boldsymbol{v}$ exists a.e. on $\Gamma$. We suppose that the body is clamped on part $\Gamma_{1}$, and the body may come in contact with an obstacle over the potential contact surface $\Gamma_{3}$. We also put $Q=\Omega \times(0, T), \Sigma=\Gamma \times(0, T), \Sigma_{1}=\Gamma_{1} \times(0, T)$, $\Sigma_{2}=\Gamma_{2} \times(0, T)$, and $\Sigma_{3}=\Gamma_{3} \times(0, T)$. We often do not indicate explicitly the dependence of functions on the spatial variable $x \in \Omega$.

Let $\mathbb{S}^{d}$ denote the space of $d \times d$ symmetric matrices. The canonical inner products and norms on $\mathbb{R}^{d}$ and $\mathbb{S}^{d}$ are given by

$$
\boldsymbol{u} \cdot \boldsymbol{v}=u_{i} v_{i}, \quad\|\boldsymbol{v}\|=(\boldsymbol{v} \cdot \boldsymbol{v})^{1 / 2} \text { for all } \boldsymbol{u}, \boldsymbol{v} \in \mathbb{R}^{d},
$$




$$
\boldsymbol{\sigma}: \boldsymbol{\tau}=\sigma_{i j} \tau_{i j}, \quad\|\boldsymbol{\tau}\|=(\boldsymbol{\tau}: \boldsymbol{\tau})^{1 / 2} \text { for all } \boldsymbol{\sigma}, \boldsymbol{\tau} \in \mathbb{S}^{d} .
$$

In what follows, we always adopt the summation convention over repeated indices.

Moreover, for a vector $\xi \in \mathbb{R}^{d}$, the normal and tangential components of $\boldsymbol{\xi}$ on the boundary are denoted by $\xi_{v}=\boldsymbol{\xi} \cdot \boldsymbol{v}$ and $\boldsymbol{\xi}_{\tau}=\boldsymbol{\xi}-\xi_{v} \boldsymbol{v}$, respectively. The normal and tangential components of the matrix $\sigma \in \mathbb{S}^{d}$ are defined on boundary by $\sigma_{v}=(\sigma \boldsymbol{v}) \cdot \boldsymbol{v}$ and $\sigma_{\tau}=\sigma \boldsymbol{v}-\sigma_{v} \boldsymbol{v}$, respectively.

We denote by $\boldsymbol{u}: Q \rightarrow \mathbb{R}^{d}$ the displacement vector, by $\boldsymbol{\sigma}: Q \rightarrow \mathbb{S}^{d}$ the stress tensor, and by $\boldsymbol{\varepsilon}(\boldsymbol{u})=\left(\varepsilon_{i j}(\boldsymbol{u})\right)$ the linearized (small) strain tensor, where $i, j=1, \ldots, d$. Recall that the components of the linearized strain tensor are given by $\boldsymbol{\varepsilon}(\boldsymbol{u})=1 / 2\left(u_{i, j}+u_{j, i}\right)$, where $u_{i, j}=\partial u_{i} / \partial x_{j}$.

The classical formulation of the contact problem reads as follows.

Problem $\mathcal{P}$ Find a displacement field $\boldsymbol{u}: Q \rightarrow \mathbb{R}^{d}$ and a stress field $\boldsymbol{\sigma}: Q \rightarrow \mathbb{S}^{d}$ such that

$$
\begin{aligned}
\operatorname{Div} \boldsymbol{\sigma}(t)+\boldsymbol{f}_{0}(t)=0 & \text { in } Q, \\
\boldsymbol{u}(t)=\mathscr{A} \boldsymbol{\varepsilon}\left(\boldsymbol{u}^{\prime}(t)\right)+\mathscr{B} \boldsymbol{\varepsilon}(\boldsymbol{u}(t))+\int_{0}^{t} \mathscr{C}(t-s) \boldsymbol{\varepsilon}(\boldsymbol{u}(s)) d s & \text { in } Q, \\
\boldsymbol{\sigma}(t) \boldsymbol{v}=\boldsymbol{f}_{N}(t) & \text { on } \Sigma_{1}, \\
-\sigma_{v}(t) \in \partial j_{v}\left(u_{v}(t)\right) & \text { on } \Sigma_{2}, \\
-\boldsymbol{\sigma}_{\tau}(t) \in \partial j_{\tau}\left(\boldsymbol{u}_{\tau}(t)\right) & \text { on } \Sigma_{3}, \\
\boldsymbol{u}(0)=\boldsymbol{u}_{0} & \text { in } \Omega .
\end{aligned}
$$

The relation (40) represents the equilibrium equation in which "Div" denotes the divergence operator for tensor-valued functions defined by $\operatorname{Div} \sigma=\left(\sigma_{i j, j}\right)$. Equation (41) is the viscoelastic constitutive law with long memory, where $\mathscr{A}$ and $\mathscr{B}$ are linear viscosity and elasticity operators, and $\mathscr{C}$ denotes the relaxation operator. Next, conditions (42) and (43) represent the displacement and the traction boundary conditions. The multivalued relations (44) and (45) are the contact and friction conditions, respectively, in which $\partial j_{\nu}$ and $\partial j_{\tau}$ denote the Clarke generalized gradients of prescribed locally Lipschitz functions $j_{v}$ and $j_{\tau}$. Finally, condition (46) represents the initial condition where $\boldsymbol{u}_{0}$ denotes the initial displacement. For concrete examples of boundary conditions (44) and (45), we refer to [9, 14, 23, 31-33].

Subsequently, we introduce the spaces needed for the variational formulation. Let $V$ be a closed subspace of $H^{1}\left(\Omega ; \mathbb{R}^{d}\right)$ defined by

$$
V=\left\{\boldsymbol{v} \in H^{1}\left(\Omega ; \mathbb{R}^{d}\right) \mid \boldsymbol{v}=0 \text { on } \Gamma_{1}\right\}
$$

and $H=L^{2}\left(\Omega ; \mathbb{R}^{d}\right)$. Then $\left(V, H, V^{*}\right)$ forms an evolution triple of spaces. Moreover, the trace operator is denoted by $\gamma: V \rightarrow L^{2}\left(\Gamma ; \mathbb{R}^{d}\right)$. Given an element $\boldsymbol{v} \in V$, 
we use the same notation $\boldsymbol{v}$ for the trace of $\boldsymbol{v}$ on the boundary. The space $V$ is equipped with the inner product and the corresponding norm given by

$$
\langle\boldsymbol{u}, \boldsymbol{v}\rangle_{V}=\langle\boldsymbol{\varepsilon}(\boldsymbol{u}), \boldsymbol{\varepsilon}(\boldsymbol{v})\rangle_{\mathcal{H}}, \quad\|\boldsymbol{v}\|=\|\boldsymbol{\varepsilon}(\boldsymbol{v})\|_{\mathcal{H}} \text { for } \boldsymbol{u}, \boldsymbol{v} \in V,
$$

where $\mathcal{H}=L^{2}\left(\Omega ; \mathbb{S}^{d}\right)$. Since $m\left(\Gamma_{1}\right)>0$, from the Korn inequality $\|\boldsymbol{v}\|_{H^{1}\left(\Omega ; \mathbb{R}^{d}\right)} \leq$ $c\|\boldsymbol{\varepsilon}(\boldsymbol{v})\|_{\mathcal{H}}$ for $\boldsymbol{v} \in V$ with $c>0$, it follows that $\|\cdot\|_{H^{1}\left(\Omega ; \mathbb{R}^{d}\right)}$ and $\|\cdot\|$ are equivalent norms on $V$. In addition, we denote by $\mathcal{Q}_{\infty}$ the space of fourth-order tensor fields given by

$$
\mathcal{Q}_{\infty}=\left\{\mathcal{E}=\left(\mathcal{E}_{i j k l}\right) \mid \mathcal{E}_{i j k l}=\mathcal{E}_{j i k l}=\mathcal{E}_{k l i j} \in L^{\infty}(\Omega), 1 \leq i, j, k, l \leq d\right\} .
$$

We assume that the viscosity and elasticity tensors have the usual properties of ellipticity and symmetry.

$\underline{H(\mathscr{A})}: \mathscr{A}: \Omega \times \mathbb{S}^{d} \rightarrow \mathbb{S}^{d}$ is a viscosity tensor, $\mathscr{A}=\left(a_{i j k l}\right) \in \mathcal{Q}_{\infty}$ such that there exists $m_{1}>0$ satisfying $\mathscr{A} \tau \cdot \tau \geq m_{1}\|\tau\|_{\mathbb{S}^{d}}^{2}$ for all $\tau \in \mathbb{S}^{d}$, a.e. in $\Omega$.

$H(\mathscr{B}): \mathscr{B}: \Omega \times \mathbb{S}^{d} \rightarrow \mathbb{S}^{d}$ is an elasticity tensor, $\mathscr{B}=\left(b_{i j k l}\right) \in \mathcal{Q}_{\infty}$ such that there exists $m_{2}>0$ satisfying $\mathscr{B} \tau \cdot \tau \geq m_{2}\|\tau\|_{\mathbb{S}^{d}}^{2}$ for all $\tau \in \mathbb{S}^{d}$, a.e. in $\Omega$.

$\underline{H(\mathscr{C})}: \mathscr{C}:[0, T] \rightarrow \mathcal{Q}_{\infty}$ is Lipschitz continuous with constant $L_{\mathscr{C}}>0$.

The body forces, surface tractions, and initial displacement satisfy $H(f): \boldsymbol{f}_{0} \in L^{2}\left(0, T ; L^{2}\left(\Omega ; \mathbb{R}^{d}\right)\right), \boldsymbol{f}_{N} \in L^{2}\left(0, T ; L^{2}\left(\Gamma_{2} ; \mathbb{R}^{d}\right)\right), \boldsymbol{u}_{0} \in V$.

The superpotentials satisfy

$H\left(j_{v}\right): \quad j_{v}: \Gamma_{3} \times \mathbb{R} \rightarrow \mathbb{R}$ is a function such that

(i) $j_{v}(\cdot, r)$ is measurable for all $r \in \mathbb{R}, j_{v}(\cdot, 0) \in L^{1}\left(\Gamma_{3}\right)$,

(ii) $j_{v}(\boldsymbol{x}, \cdot)$ is locally Lipschitz for a.e. $\boldsymbol{x} \in \Gamma_{3}$,

(iii) $\left|\partial j_{v}(\boldsymbol{x}, r)\right| \leq c_{v}(1+|r|)$ for a.e. $\boldsymbol{x} \in \Gamma_{3}$, all $r \in \mathbb{R}$ with $c_{v}>0$,

(iv) $\left(\eta_{1}-\eta_{2}\right)\left(r_{1}-r_{2}\right) \geq-m_{v}\left|r_{1}-r_{2}\right|^{2}$ for all $\eta_{i} \in \partial j_{v}\left(\boldsymbol{x}, r_{i}\right), r_{i} \in \mathbb{R}, i=1,2$ for a.e. $\boldsymbol{x} \in \Gamma_{3}$ with $m_{v}>0$.

$\underline{H\left(j_{\tau}\right)}: \quad j_{\tau}: \Gamma_{3} \times \mathbb{R}^{d} \rightarrow \mathbb{R}$ is a function such that

(i) $j_{\tau}(\cdot, \boldsymbol{\xi})$ is measurable for all $\boldsymbol{\xi} \in \mathbb{R}^{d}, j_{\tau}(\cdot, \mathbf{0}) \in L^{1}\left(\Gamma_{3}\right)$,

(ii) $j_{\tau}(\boldsymbol{x}, \cdot)$ is locally Lipschitz for a.e. $\boldsymbol{x} \in \Gamma_{3}$,

(iii) $\left\|\partial j_{\tau}(\boldsymbol{x}, \boldsymbol{\xi})\right\|_{\mathbb{R}^{d}} \leq c_{\tau}\left(1+\|\boldsymbol{\xi}\|_{\mathbb{R}^{d}}\right.$ ) for a.e. $\boldsymbol{x} \in \Gamma_{3}$, all $\boldsymbol{\xi} \in \mathbb{R}^{d}$ with $c_{\tau}>0$,

(iv) $\left(\boldsymbol{\eta}_{1}-\boldsymbol{\eta}_{2}\right) \cdot\left(\boldsymbol{\xi}_{1}-\boldsymbol{\xi}_{2}\right) \geq-m_{\tau}\left\|\boldsymbol{\xi}_{1}-\boldsymbol{\xi}_{2}\right\|^{2}$ for all $\boldsymbol{\eta}_{i} \in \partial j_{\tau}\left(\boldsymbol{x}, \boldsymbol{\xi}_{i}\right), \boldsymbol{\xi}_{i} \in \mathbb{R}^{d}, i=1,2$ for a.e. $\boldsymbol{x} \in \Gamma_{3}$ with $m_{\tau}>0$.

In the hypotheses $H\left(j_{v}\right)$ and $H\left(j_{\tau}\right)$, the subdifferential is taken with respect to the last variables of $j_{\nu}$ and $j_{\tau}$, respectively.

Next, we define the operators $A, B \in \mathcal{L}\left(V, V^{*}\right)$ by

$$
\langle A \boldsymbol{u}, \boldsymbol{v}\rangle_{V^{*} \times V}=\langle\mathscr{A} \boldsymbol{\varepsilon}(\boldsymbol{u}), \boldsymbol{\varepsilon}(\boldsymbol{v})\rangle_{\mathcal{H}}, \quad\langle B \boldsymbol{u}, \boldsymbol{v}\rangle_{V^{*} \times V}=\langle\mathscr{B} \boldsymbol{\varepsilon}(\boldsymbol{u}), \boldsymbol{\varepsilon}(\boldsymbol{v})\rangle_{\mathcal{H}}
$$

for $\boldsymbol{u}, \boldsymbol{v} \in V$, and the operator $\mathcal{R}: \mathcal{V} \rightarrow \mathcal{V}^{*}$ by

$$
\langle(\mathcal{R} \boldsymbol{w})(t), \boldsymbol{v}\rangle_{V^{*} \times V}=\left\langle\int_{0}^{t} \mathscr{C}(t-s) \boldsymbol{\varepsilon}(\boldsymbol{w}(s)) d s, \boldsymbol{\varepsilon}(\boldsymbol{v})\right\rangle_{\mathcal{H}}
$$

for all $w \in \mathcal{V}, v \in V$, a.e. $t \in(0, T)$. 
To obtain the weak formulation of the problem (40)-(46), we assume the sufficient smoothness of the functions involved, use the equilibrium equation (40) and the Green formula. We obtain

$$
\langle\boldsymbol{\sigma}(t), \boldsymbol{\varepsilon}(\boldsymbol{v}))_{\mathcal{H}}=\left\langle\boldsymbol{f}_{0}(t), \boldsymbol{v}\right\rangle_{H}+\int_{\Gamma} \boldsymbol{\sigma}(t) \boldsymbol{v} \cdot \boldsymbol{v} d \Gamma
$$

for $v \in V$. Taking into account the boundary condition (42) and (43), we have

$$
\langle\boldsymbol{\sigma}(t), \boldsymbol{\varepsilon}(\boldsymbol{v})\rangle_{\mathcal{H}}-\int_{\Gamma_{3}} \boldsymbol{\sigma}(t) \boldsymbol{v} \cdot \boldsymbol{v} d \Gamma=\langle\boldsymbol{f}(t), \boldsymbol{v}\rangle,
$$

where $\boldsymbol{f} \in \mathcal{V}^{*}$ is given by $\langle\boldsymbol{f}(t), \boldsymbol{v}\rangle=\left\langle\boldsymbol{f}_{0}(t), \boldsymbol{v}\right\rangle_{H}+\left\langle\boldsymbol{f}_{N}(t), \boldsymbol{v}\right\rangle_{L^{2}\left(\Gamma_{2} ; \mathbb{R}^{d}\right)}$ for $\boldsymbol{v} \in V$. On the other hand, by the ortogonality relation (cf. (6.33) in [23]), we get

$$
\int_{\Gamma_{3}} \boldsymbol{\sigma}(t) \boldsymbol{v} \cdot \boldsymbol{v} d \Gamma=\int_{\Gamma_{3}}\left(\sigma_{\nu}(t) v_{\nu}+\boldsymbol{\sigma}_{\tau}(t) \cdot \boldsymbol{v}_{\tau}\right) d \Gamma .
$$

The contact and friction boundary conditions (44) and (45) can be equivalently formulated as follows

$$
-\sigma_{v}(t) r \leq j_{v}^{0}\left(u_{v} ; r\right) \text { for all } r \in \mathbb{R}, \quad-\boldsymbol{\sigma}_{\tau}(t) \cdot \boldsymbol{\xi} \leq j_{\tau}^{0}\left(\boldsymbol{u}_{\tau} ; \boldsymbol{\xi}\right) \text { for all } \boldsymbol{\xi} \in \mathbb{R}^{d} .
$$

Using (41), (48), (51), and (52), from (50), we obtain the following hemivariational inequality which is a weak formulation of the problem (40)-(46): find $\boldsymbol{u}:(0, T) \rightarrow V$ such that $\boldsymbol{u}, \boldsymbol{u}^{\prime} \in \mathcal{V}$ and

$$
\left\{\begin{array}{l}
\left\langle A \boldsymbol{u}^{\prime}(t)+B \boldsymbol{u}(t)+(\mathcal{R} \boldsymbol{u})(t), \boldsymbol{v}\right\rangle+\int_{\Gamma_{3}}\left(j_{v}^{0}\left(u_{v} ; v_{v}\right)+j_{\tau}^{0}\left(\boldsymbol{u}_{\tau} ; \boldsymbol{v}_{\tau}\right)\right) d \Gamma \\
\quad \geq\langle\boldsymbol{f}(t), \boldsymbol{v}\rangle \text { for all } \boldsymbol{v} \in V, \text { a.e. } t \in(0, T), \\
\boldsymbol{u}(0)=\boldsymbol{u}_{0} .
\end{array}\right.
$$

\subsection{Existence and uniqueness for contact problem}

Let $X=L^{2}\left(\Gamma_{3} ; \mathbb{R}^{d}\right)$ and consider the functional $J: X \rightarrow \mathbb{R}$ defined by

$$
J(v)=\int_{\Gamma_{3}}\left(j_{v}\left(\boldsymbol{x}, v_{v}(\boldsymbol{x})\right)+j_{\tau}\left(\boldsymbol{x}, \boldsymbol{v}_{\tau}(\boldsymbol{x})\right)\right) d \Gamma \text { for all } \boldsymbol{v} \in X .
$$

Following [25, Theorem 5.1] and [23, Corollary 4.15], we recall the following properties of the functional $J$.

Lemma 14 Under the hypotheses $H\left(j_{v}\right)$ and $H\left(j_{\tau}\right)$, if, in addition,

$$
\left\{\begin{array}{l}
\text { either } j_{v}(\boldsymbol{x}, \cdot) \text { or }-j_{v}(\boldsymbol{x}, \cdot) \text { is regular and } \\
\text { either } j_{\tau}(\boldsymbol{x}, \cdot) \text { or }-j_{\tau}(\boldsymbol{x}, \cdot) \text { is regular }
\end{array}\right.
$$

then the functional $J$ defined by (54) satisfies 
(i) $J$ is Lipschitz continuous on bounded subsets of $X$,

(ii) $\|\partial J(\boldsymbol{v})\|_{X^{*}} \leq c_{1}\left(1+\|\boldsymbol{v}\|_{X}\right)$ for all $\boldsymbol{v} \in X$ with $c_{1}=\max \left\{c_{\tau}, c_{\nu}\right\}$,

(iii) for all $\boldsymbol{v}, \boldsymbol{w} \in X, \boldsymbol{\xi} \in \partial J(\boldsymbol{v})$ and $\boldsymbol{\eta} \in \partial J(\boldsymbol{w})$, we have

$$
\langle\boldsymbol{\xi}-\boldsymbol{\eta}, \boldsymbol{v}-\boldsymbol{w}\rangle_{X^{*} \times X} \geq-m_{3}\|\boldsymbol{v}-\boldsymbol{w}\|_{X}^{2}
$$

with $m_{3}=m_{v}+m_{\tau}$,

(iv) for all $\boldsymbol{v}, \boldsymbol{w} \in X$, we have

$$
J^{0}(\boldsymbol{v} ; \boldsymbol{w})=\int_{\Gamma_{3}}\left(j_{v}^{0}\left(v_{\nu} ; w_{\nu}\right)+j_{\tau}^{0}\left(\boldsymbol{v}_{\tau} ; \boldsymbol{w}_{\tau}\right)\right) d \Gamma
$$

where $J^{0}(\boldsymbol{v} ; \boldsymbol{w})$ denotes the directional derivative of $J$ at a point $\boldsymbol{v} \in X$ in the direction $\boldsymbol{w} \in X$.

Under our notation, we associate with the hemivariational inequality (53) the following inclusion: find $\boldsymbol{u} \in \mathcal{V}$ such that $\boldsymbol{u}^{\prime} \in \mathcal{V}$ and

$$
\left\{\begin{array}{l}
\left\langle A \boldsymbol{u}^{\prime}(t)+B \boldsymbol{u}(t)+(\mathcal{R} \boldsymbol{u})(t)-\boldsymbol{f}(t), \boldsymbol{v}\right\rangle+J^{0}(\gamma \boldsymbol{u}(t) ; \gamma \boldsymbol{v}) \geq 0 \\
\boldsymbol{u}(0)=\boldsymbol{u}_{0} .
\end{array}\right.
$$

Note that if the hypotheses $H\left(j_{v}\right)$ and $H\left(j_{\tau}\right)$ hold, then every solution to (58) is a solution to (53). The converse holds provided $j_{v}$ and $j_{\tau}$ satisfy the regularity condition (55). These facts follow from the definition of the Clarke generalized gradient and Lemma 14.

The existence, uniqueness, and regularity result for the hemivariational inequality (53) is given in the following result.

Theorem 15 If the hypotheses $H(\mathscr{A}), H(\mathscr{B}), H(\mathscr{C}), H(f), H\left(j_{v}\right)$, and $H\left(j_{\tau}\right)$ and regularity condition (55) hold and the inequality $m_{2}>\left(m_{v}+m_{\tau}\right)\|\gamma\|^{2}$ is satisfied, then problem (53) has a unique solution $\boldsymbol{u} \in H^{1}(0, T ; V)$.

Proof It follows from $H(\mathscr{A})$ and $H(\mathscr{B})$ that the operators $A$ and $B$ defined by (48) satisfy $H(A)$ with $m_{A}=m_{1}$ and $H(B)$ with $m_{B}=m_{2}$, respectively. It is obvious from the definition of $\mathcal{R}$ (see (49)) and hypothesis $H(\mathscr{C})$ that $H(E)$ and $H(q)$ are satisfied with $E=I$ and $q=\mathscr{C}$. Moreover, we put $M=\gamma \in \mathcal{L}(V, X), \gamma$ is the trace operator. It is a consequence of Lemma 14 that the functional $J$ given by (54) satisfies $H(J)$ with $c_{J}=c_{1}$ and $m_{J}=m_{3}$ (see Lemma 14). Also $H(M)$ follows easily by the properties of the trace operator. The conclusion is a consequence of Theorem 11, which completes the proof of this theorem.

We say that a couple of functions $(\boldsymbol{u}, \boldsymbol{\sigma})$ which satisfies (41) and (53) is called a weak solution to Problem $\mathcal{P}$. We conclude that, under the assumptions of Theorem 15, Problem $\mathcal{P}$ has a unique weak solution. Moreover, the weak solution has the following regularity $\boldsymbol{u} \in H^{1}(0, T ; V), \boldsymbol{\sigma} \in L^{2}\left(0, T ; L^{2}\left(\Omega, \mathbb{S}^{d}\right)\right)$, and $\operatorname{Div} \boldsymbol{\sigma} \in \mathcal{V}^{*}$. 


\subsection{Numerical analysis of contact problem}

In this section, we will apply the results from Section 5 to establish an optimal order error estimate for the fully discrete solution of the contact problem in Problem $\mathcal{P}$. Here, we consider the frictionless boundary condition on $\Gamma_{3}$, i.e., the frictional boundary (45) will be reduced to

$$
\boldsymbol{\sigma}_{\tau}(t)=\mathbf{0} \text { on } \Sigma_{3} .
$$

In addition, without loss of generality, we may assume that $\boldsymbol{u}_{0}=\mathbf{0}$. We use the same the spaces as introduced in Section 6.1. Then, consider the trace operator $\gamma: V \rightarrow$ $L^{2}\left(\Gamma_{3} ; \mathbb{R}^{d}\right)$. It follows from the Sobolev trace theorem that

$$
\|\gamma \boldsymbol{v}\|_{L^{2}\left(\Gamma_{3} ; \mathbb{R}^{d}\right)} \leq c_{0}\|\boldsymbol{v}\|_{V} \text { for all } \boldsymbol{v} \in V
$$

for some constant $c_{0}>0$, which depends only on $\Omega, \Gamma_{1}$, and $\Gamma_{3}$. Let $X=L^{2}\left(\Gamma_{3}\right)$ and define the operators $\gamma_{v}: L^{2}\left(\Gamma_{3} ; \mathbb{R}^{d}\right) \rightarrow X, \gamma_{v} \boldsymbol{v}=v_{v}$ for $\boldsymbol{v} \in L^{2}\left(\Gamma_{3} ; \mathbb{R}^{d}\right)$, and $M=\gamma_{\nu} \circ \gamma: V \rightarrow X$. We also consider the functional $J: X \rightarrow \mathbb{R}$ defined by

$$
J(v)=\int_{\Gamma_{3}} j_{v}\left(\boldsymbol{x}, v_{v}(\boldsymbol{x})\right) d \Gamma \text { for all } v \in X .
$$

If either $j_{v}(\boldsymbol{x}, \cdot)$ or $-j_{v}(\boldsymbol{x}, \cdot)$ is regular and $H\left(j_{v}\right)$ holds, then by Lemma 14, (48) and (49), the contact problem (40)-(46) with $j_{\tau}=0$ has following equivalent variational formulation.

Problem 16 Find $\boldsymbol{u} \in \mathcal{V}$ such that $\boldsymbol{u}^{\prime} \in \mathcal{V}$ and

$$
\left\{\begin{array}{l}
\left\langle A \boldsymbol{u}^{\prime}(t)+B \boldsymbol{u}(t)+(\mathcal{R} \boldsymbol{u})(t)-\boldsymbol{f}(t), \boldsymbol{v}-\boldsymbol{u}(t)\right\rangle \\
\quad+J^{0}(\gamma \boldsymbol{u}(t) ; \gamma \boldsymbol{v}-\gamma \boldsymbol{u}(t)) \geq 0 \text { for all } \boldsymbol{v} \in V, \text { a.e. } t \in(0, T) \\
\boldsymbol{u}(0)=\mathbf{0}
\end{array}\right.
$$

From Theorem 15, we deduce that under the hypotheses $H(\mathscr{A}), H(\mathscr{B}), H(\mathscr{C})$, and $H(f), H\left(j_{v}\right)$. If either $j_{v}(\boldsymbol{x}, \cdot)$ or $-j_{v}(\boldsymbol{x}, \cdot)$ is regular and the inequality $m_{2}>$ $\left(m_{v}+m_{\tau}\right)\|\gamma\|^{2}$ hold, then Problem 16 has a unique solution $\boldsymbol{u} \in H^{1}(0, T ; V)$.

Next, we pass to the numerical approximation of Problem 16. Likewise, in Section 5, for an integer $N>0$, let $k=\frac{T}{N}$ be the time step length. For simplicity, we suppose that $\Omega$ is a polygonal/polyhedral domain and express the three parts of the boundary, $\Gamma_{k}, k=1,2,3$, as a union of closed flat components with disjoint interiors

$$
\bar{\Gamma}_{j}=\cup_{i=1}^{i_{j}} \Gamma_{j, i}, \quad 1 \leq j \leq 3 .
$$

Subsequently, we consider a regular family of meshes $\left\{\mathcal{T}^{h}\right\}$ that partition $\bar{\Omega}$ into triangles/tetrahedrons compatible with the splitting of the boundary $\partial \Omega$ into $\Gamma_{j, i}$, $1 \leq i \leq i_{j}, 1 \leq j \leq 3$. This means that if the intersection of one side/face of an element with one set $\Gamma_{j, i}$ has a positive measure with respect to $\Gamma_{j, i}$, then the side/face lies entirely in $\Gamma_{j, i}$. Corresponding to the family $\left\{\mathcal{T}^{h}\right\}$, we define the linear element space

$$
V^{h}=\left\{\boldsymbol{v}^{h} \in C\left(\bar{\Omega} ; \mathbb{R}^{d}\right)\left|\boldsymbol{v}^{h}\right|_{U} \in \mathbb{P}_{1}(U)^{d}, U \in \mathcal{T}^{h}, \boldsymbol{v}^{h}=0 \text { on } \Gamma_{1}\right\},
$$


where $\mathbb{P}_{1}(U)^{d}$ denotes a set of all linear functions whose domain of definition is $U$ (cf. [16, p. 70]).

Now, we are in a position to formulate the following fully discrete approximation problem for Problem 16.

Problem 17 Find $\boldsymbol{u}^{h k}=\left\{\boldsymbol{u}_{n}^{h k}\right\} \subset V^{h}$ such that $\boldsymbol{u}_{0}^{h k}=\mathbf{0}$ and

$$
\left\{\begin{array}{c}
\left\langle A \delta \boldsymbol{u}_{n}^{h k}+B \boldsymbol{u}_{n}^{h k}+\mathcal{R}_{n}^{k} \boldsymbol{u}^{h k}, \boldsymbol{v}^{h}-\boldsymbol{u}_{n}^{h k}\right\rangle+J^{0}\left(M \boldsymbol{u}_{n}^{h k} ; M \boldsymbol{v}^{h}-M \boldsymbol{u}_{n}^{h k}\right) \\
\geq\left\langle\boldsymbol{f}_{n}, \boldsymbol{v}^{h}-\boldsymbol{u}_{n}^{h k}\right\rangle \text { for all } \boldsymbol{v}^{h} \in V^{h}
\end{array}\right.
$$

for all $n=1,2, \ldots, N$.

In the sequel, we assume that the solution of Problem 16 has the following additional regularity

$$
\left\{\begin{array}{l}
\boldsymbol{u} \in H^{1}\left(0, T ; H^{2}(\Omega)\right), \boldsymbol{u}^{\prime \prime} \in L^{2}(0, T ; V), \\
\left.u_{v}\right|_{\Gamma_{3, i}} \in C\left(0, T ; H^{2}\left(\Gamma_{3, i}\right)\right),\left.\quad \sigma_{v}\right|_{\Gamma_{3, i}} \in C\left(0, T ; L^{2}\left(\Gamma_{3, i}\right)\right)
\end{array}\right.
$$

for $1 \leq i \leq i_{3}$. Then, the function $(t, \boldsymbol{x}) \rightarrow \boldsymbol{u}(t, \boldsymbol{x})$ is continuous. This means that the pointwise values of $\boldsymbol{u}$ are well-defined. So, take $\boldsymbol{v}_{n}^{h}=\Pi^{h} \boldsymbol{u}_{n} \in V^{h}$ to be the finite element interpolant of $\boldsymbol{u}_{n}(\boldsymbol{x})=\boldsymbol{u}\left(t_{n}, \boldsymbol{x}\right)$, where $\Pi^{h} \boldsymbol{u}_{n}$ denotes the piecewise constant Lagrange interpolation of $\boldsymbol{u}_{n}$ (cf. [16, p. 122]). We use the Céa-type inequality (13) to get

$$
\begin{aligned}
& \max _{1 \leq n \leq N}\left\|\boldsymbol{u}_{n}-\boldsymbol{u}_{n}^{h k}\right\|_{V}^{2} \leq C\left[k^{2}+\max _{1 \leq n \leq N}\left\|\boldsymbol{u}_{n}-\Pi^{h} \boldsymbol{u}_{n}\right\|_{V}^{2}+k \sum_{n=1}^{N}\left(\left\|\delta\left(\boldsymbol{u}_{n}-\Pi^{h} \boldsymbol{u}_{n}\right)\right\|_{V}^{2}\right.\right. \\
& \left.\left.\quad+\left\|u_{n, v}-\Pi^{h} u_{n, v}\right\|_{X}+\left|S_{l}\left(\Pi^{h} \boldsymbol{u}_{n}\right)\right|+\left\|\boldsymbol{\delta}_{n}\right\|_{V}^{2}\right)\right],
\end{aligned}
$$

where

$$
\begin{aligned}
& \boldsymbol{\delta}_{n}=\delta \boldsymbol{u}_{n}-\boldsymbol{u}_{n}^{\prime}, \\
& S_{n}(\boldsymbol{v})=\left\langle A \boldsymbol{u}_{n}^{\prime}+B \boldsymbol{u}_{n}+\mathcal{R}_{n} \boldsymbol{u}-\boldsymbol{f}_{n}, \boldsymbol{v}-\boldsymbol{u}_{n}\right\rangle+J^{0}\left(M \boldsymbol{u}_{n} ; M \boldsymbol{v}-M \boldsymbol{u}_{n}\right) .
\end{aligned}
$$

It follows from [13, Lemma 11.5] that

$$
\left\|\boldsymbol{\delta}_{n}\right\|_{V} \leq\left\|\boldsymbol{u}^{\prime \prime}\right\|_{L^{1}\left(t_{n-1}, t_{n} ; V\right)}
$$

This together with Hölder inequality implies that

$$
\left\|\boldsymbol{\delta}_{n}\right\|_{V}^{2} \leq k\left\|\boldsymbol{u}^{\prime \prime}\right\|_{L^{2}\left(t_{n-1}, t_{n} ; V\right)}^{2}
$$

and

$$
k \sum_{n=1}^{N}\left\|\boldsymbol{\delta}_{n}\right\|_{V}^{2} \leq k^{2}\left\|\boldsymbol{u}^{\prime \prime}\right\|_{L^{2}(0, T ; V)}^{2}
$$

Next, we use the fact

$$
\delta\left(\boldsymbol{u}_{n}-\Pi^{h} \boldsymbol{u}_{n}\right)=\frac{1}{k} \int_{t_{n-1}}^{t_{n}}\left(\boldsymbol{u}^{\prime}(s)-\Pi^{h} \boldsymbol{u}^{\prime}(s)\right) d s
$$


to obtain

$$
\left\|\delta\left(\boldsymbol{u}_{n}-\Pi^{h} \boldsymbol{u}_{n}\right)\right\|_{V}^{2} \leq \frac{1}{k} \int_{t_{n-1}}^{t_{n}}\left\|\boldsymbol{u}^{\prime}(s)-\Pi^{h} \boldsymbol{u}^{\prime}(s)\right\|_{V}^{2} d s .
$$

Hence, we have

$$
k \sum_{n=1}^{N}\left\|\delta\left(\boldsymbol{u}_{n}-\Pi^{h} \boldsymbol{u}_{n}\right)\right\|_{V}^{2} \leq \int_{0}^{T}\left\|\boldsymbol{u}^{\prime}(s)-\Pi^{h} \boldsymbol{u}^{\prime}(s)\right\|_{V}^{2} d s
$$

and

$$
k \sum_{n=1}^{N}\left\|\delta\left(\boldsymbol{u}_{n}-\Pi^{h} \boldsymbol{u}_{n}\right)\right\|_{V}^{2} \leq C h^{2}\left\|\boldsymbol{u}^{\prime}\right\|_{L^{2}(0, T ; V)}^{2} .
$$

Recall that $\Pi^{h} u_{n, v}$ is the finite element interpolant of $u_{n, v}$ on each component $\Gamma_{3, i}$. Combining (66) with the hypothesis (63), we get

$$
k \sum_{n=1}^{N}\left\|u_{n, v}-\Pi^{h} u_{n, v}\right\|_{X} \leq C h^{2} \sum_{i=1}^{i_{3}}\left\|u_{\nu}\right\|_{L^{\infty}\left(0, T ; H^{2}\left(\Gamma_{3, i}\right)\right)} .
$$

On the other hand, we estimate the residual quantity $\left|S_{n}(\boldsymbol{v})\right|$. To this end, we use the fact (see (50), (51), and (59)) that

$$
\left\langle A \boldsymbol{u}_{n}^{\prime}+B \boldsymbol{u}_{n}+\mathcal{R}_{n} \boldsymbol{u}-\boldsymbol{f}_{n}, \boldsymbol{v}\right\rangle=\int_{\Gamma_{3}} \sigma_{\nu}(t) v_{v} d \Gamma \text { for all } \boldsymbol{v} \in V
$$

to get

$$
S_{n}(\boldsymbol{v})=\int_{\Gamma_{3}}\left(\sigma_{n, v}+\xi_{n}\right)\left(\Pi^{h} u_{n, v}-u_{n, v}\right) d \Gamma
$$

for some $\xi_{n} \in \partial J\left(M \boldsymbol{u}_{n}\right)$. This implies

$$
\left|S_{n}\left(\Pi^{h} \boldsymbol{u}_{n}\right)\right| \leq C\left\|\Pi^{h} u_{n, v}-u_{n, v}\right\|_{X},
$$

and, therefore, we have

$$
k \sum_{n=1}^{N}\left|S_{n}\left(\Pi^{h} \boldsymbol{u}_{n}\right)\right| \leq C h^{2} \sum_{i=1}^{i_{3}}\left\|u_{v}\right\|_{L^{\infty}\left(0, T ; H^{2}\left(\Gamma_{3, i}\right)\right)} .
$$

This estimate together with (65)-(67) implies the following optimal estimate for the fully discrete scheme (62).

Theorem 18 Assume that $\boldsymbol{u}$ and $\boldsymbol{u}^{h k}$ are solutions to Problems 16 and 17, respectively, and the regularity condition (63) holds. Then, we have

$$
\max _{1 \leq n \leq N}\left\|\boldsymbol{u}_{n}-\boldsymbol{u}_{n}^{h k}\right\|_{V} \leq C(k+h),
$$

where $C>0$ is independent of $k$ and $h$.

In the optimal error estimate of Theorem 18, the method is of first order in spatial mesh size and in the time step. 
Funding information This project was supported by the H2020-MSCA-RISE-2018 Research and Innovation Staff Exchange Scheme Fellowship within the Project No. 823731 CONMECH, the National Science Center of Poland under Maestro Project No. UMO-2012/06/A/ST1/00262, and National Science Center of Poland under Preludium Project No. 2017/25/N/ST1/00611. It is also supported by the International Project co-financed by the Ministry of Science and Higher Education of Republic of Poland under Grant No. 3792/GGPJ/H2020/2017/0, Natural Sciences Foundation of Guangxi Grant No. 2018JJA110006, Beibu Gulf University Project No. 2018KYQD06, National Natural Science Foundation of China (Grant Nos. 11561007).

Open Access This article is distributed under the terms of the Creative Commons Attribution 4.0 International License (http://creativecommons.org/licenses/by/4.0/), which permits unrestricted use, distribution, and reproduction in any medium, provided you give appropriate credit to the original author(s) and the source, provide a link to the Creative Commons license, and indicate if changes were made.

Publisher's note Springer Nature remains neutral with regard to jurisdictional claims in published maps and institutional affiliations.

\section{References}

1. Alleche, B.B., Rădulescu, V.D.: The Ekeland variational principle for equilibrium problems revisited and applications. Nonlin. Anal. Real World Appl. 23, 17-25 (2015)

2. Aubin, J.P., Cellina, A.: Differential Inclusions. Set-Valued Maps and Viability Theory. Springer, Berlin (1984)

3. Bartosz, K.: Numerical methods for evolution hemivariational inequalities, Chapter 5. In: Han, W., et al (eds.) Advances in Variational and Hemivariational Inequalities with Applications. Theory, Numerical Analysis, and Applications, Advances in Mechanics and Mathematics, vol. 33, pp. 111-144. Springer (2015)

4. Carstensen, C., Gwinner, J.: A theory of discretization for nonlinear evolution inequalities applied to parabolic Signorini problems. Ann. Mat. Pura Appl. 177, 363-394 (1999)

5. Clarke, F.H.: Optimization and Nonsmooth Analysis. Wiley Interscience, New York (1983)

6. Costea, N., Rădulescu, V.D.: Inequality problems of quasi-hemivariational type involving set-valued operators and a nonlinear term. J. Glob. Optim. 52, 743-756 (2012)

7. Denkowski, Z., Migórski, S., Papageorgiou, N.S.: An Introduction to Nonlinear Analysis: Theory. Kluwer Academic/Plenum Publishers, Boston (2003)

8. Denkowski, Z., Migórski, S., Papageorgiou, N.S.: An Introduction to Nonlinear Analysis: Applications. Kluwer Academic/Plenum Publishers, Boston (2003)

9. Duvaut, G., Lions, J.L.: Inequalities in Mechanics and Physics. Springer, Berlin (1976)

10. Han, J.F., Migórski, S., Zeng, H.: Analysis of a dynamic viscoelastic unilateral contact problem with normal damped response. Nonlin. Anal. Real World Appl. 28, 229-250 (2016)

11. Han, W., Migórski, S., Sofonea, M.: Analysis of a general dynamic history-dependent variationalhemivariational inequality. Nonlin. Anal. Real World Appl. 36, 69-88 (2017)

12. Han, W., Sofonea, M., Barboteu, M.: Numerical analysis of elliptic hemivariational inequalities. SIAM J. Numer. Anal. 55, 640-663 (2017)

13. Han, W., Reddy, B.D.: Plasticity: Mathematical Theory and Numerical Analysis. Springer, 2 (2013)

14. Han, W., Sofonea, M.: Quasistatic Contact Problems in Viscoelasticity and Viscoplasticity Studies in Advanced Mathematics, vol. 30. Americal Mathematical Society, Providence, International Press, Somerville (2002)

15. Han, W., Migórski, S., Sofonea, M.: A class of variational-hemivariational inequalities with applications to frictional contact problems. SIAM J. Math. Anal. 46, 3891-3912 (2014)

16. Haslinger, J., Miettinen, M., Panagiotopoulos, P.D.: Finite Element Methods for Hemivariational Inequalities. Kluwer, Dordrecht (1999)

17. Kačur, J.: Application of Rothe's method to perturbed linear hyperbolic equations and variational inequalities. Czechoslov. Math. J. 34, 92-106 (1984)

18. Kacur, J.: Method of Rothe in Evolution Equations Teubner-Texte zur Mathematik, vol. 80. B.G. Teubner, Leipzig (1985) 
19. Liu, Z.H., Migórski, S., Zeng, S.D.: Partial differential variational inequalities involving nonlocal boundary conditions in Banach spaces. J. Differ. Equa. 263, 3989-4006 (2017)

20. Migórski, S.: Existence of solutions for a class of history-dependent evolution hemivariational inequalities. Dyn. Syst. Appl. 21, 319-330 (2012)

21. Migórski, S., Zeng, S.D.: Hyperbolic hemivariational inequalities controlled by evolution equations with application to adhesive contact model. Nonlin. Anal. Real World Appl. 43, 121-143 (2018)

22. Migórski, S., Zeng, S.D.: Penalty and regularization method for variational-hemivariational inequalities with application to frictional contact. ZAMM-Z Angew. Math. Me. 98, 1503-1520 (2018)

23. Migórski, S., Ochal, A., Sofonea, M.: Nonlinear Inclusions and Hemivariational Inequalities. Models and Analysis of Contact Problems, Advances in Mechanics and Mathematics, vol. 26. Springer, New York (2013)

24. Migórski, S., Ochal, A.: Quasti-static hemivariational inequality via vanishing acceleration approach. SIAM J. Math. Anal. 41, 1415-1435 (2009)

25. Migórski, S., Ochal, A., Sofonea, M.: Integrodifferential hemivariational inequalities with applications to viscoelastic frictional contact. Math. Models Methods Appl. Sci. 18, 271-290 (2008)

26. Migórski, S., Ochal, A., Sofonea, M.: History-dependent variational-hemivariational inequalities in contact mechanics. Nonlin. Anal. Real World Appl. 22, 604-618 (2015)

27. Migórski, S., Zeng, S.D.: A class of differential hemivariational inequalities in Banach spaces. J. Global Optim. 72, 761-779 (2018)

28. Migórski, S., Ochal, A., Sofonea, M.: Evolutionary inclusions and hemivariational inequalities, Chapter 2 in Advances in Variational and Hemivariational Inequalities. Theory, Numerical Analysis, and Applications. In: Han, W., et al (eds.) Advances in Mechanics and Mathematics Series, vol. 33, pp. 39-64. Springer, Heidelberg (2015)

29. Migórski, S., Ogorzaly, J.: A class of evolution variational inequalities with memory and its application to viscoelastic frictional contact problems. J. Math. Anal. Appl. 442, 685-702 (2016)

30. Migórski, S., Ogorzaly, J.: Dynamic history-dependent variational-hemivariational inequalities with applications to contact mechanics. Zeitschrift fü,r angewandte Mathematik und Physik 68, 15 (2017). https://doi.org/10.1007/s00033-016-0758-4

31. Naniewicz, Z., Panagiotopoulos, P.D.: Mathematical Theory of Hemivariational Inequalities and Applications. Marcel Dekker, Inc., New York (1995)

32. Panagiotopoulos, P.D.: Inequality Problems in Mechanics and Applications. Convex and Nonconvex Energy Functions. Basel, Birkhäuser (1985)

33. Panagiotopoulos, P.D.: Hemivariational Inequalities, Applications in Mechanics and Engineering. Springer, Berlin (1993)

34. Papageorgiou, N.S., Rădulescu, V.D., Repovš, D.D.: Nonhomogeneous hemivariational inequalities with indefinite potential and robin boundary condition. J. Optim. Theory Appl. 175, 293-323 (2017)

35. Shillor, M., Sofonea, M., Telega, J.J.: Models and Analysis of Quasistatic Contact. Springer, Berlin (2004)

36. Sofonea, M., Han, W., Shillor, M.: Analysis and Approximation of Contact Problems with Adhesion or Damage. Chapman \& Hall/CRC, Boca Raton (2005)

37. Sofonea, M., Han, W., Migórski, S.: Numerical analysis of history-dependent variational-hemivariational inequalities with applications to contact problems. Euro. J. Appl. Math. 26, 427-452 (2015)

38. Sofonea, M., Migórski, S., Han, W.: A penalty method for history-dependent variational-hemivariational inequalities. Comput. Math. Appl. 75, 2561-2573 (2018)

39. Sofonea, M., Matei, A.: History-dependent quasivariational inequalities arising in contact mechanics. Eur. J. Appl. Math. 22, 471-491 (2011)

40. Sofonea, M., Patrulescu, F.: Penalization of history-dependent variational inequalities. Eur. J. Appl. Math. 25, 155-176 (2014)

41. Sofonea, M., Xiao, Y.: Fully history-dependent quasivariational inequalities in contact mechanics. Appl. Anal. 95, 2464-2484 (2016)

42. Zeidler, E.: Nonlinear Functional Analysis and Applications II A/B. Springer, New York (1990)

43. Zeng, S.D., Liu, Z.H., Migórski, S.: A class of fractional differential hemivariational inequalities with application to contact problem. Z. Angew. Math. Phys. 69, 36,23 (2018)

44. Zeng, S.D.: A class of time-fractional hemivariational inequalities with application to frictional contact problem. Commun. Nonlinear Sci. 56, 34-48 (2018)

45. Zeng, S.D., Migórski, S.: Noncoercive hyperbolic variational inequalities with applications to contact mechanics. J. Math. Anal. Appl. 455, 619-637 (2017) 